\title{
Grenzüberschreitende Identitäten im badischen Oberrheingebiet: Unterschiede in der Konstruktion sprachlicher und regionaler Verbundenheit mit dem Elsass*
}

\author{
Martin Pfeiffer (Freiburg i. Br.)
}

\begin{abstract}
Based on a qualitative analysis of 127 sociolinguistic interviews with speakers of Alemannic from 22 villages and towns along the Franco-German border at the Upper Rhine in Baden (Germany), this contribution investigates the construction of trans-border identities. The paper explores how Badeners perceive the relationship with Alsace (France) with regard to three thematic fields: 1) regional ties with Alsace, 2) language choice in communication across the border, and 3) comprehension of the Alsatian dialect.

Two factors are shown to play a major role for the construction of trans-border identities. First, identities vary between regions, which can be explained by historical differences, especially with respect to political circumstances. The closer the historical relationship between the respective region and Alsace, the stronger the trans-border identity. Second, there is an influence of the geographical distance to the border. The closer a village is located to the border (the Rhine), the stronger the (self- and other-)ascription of linguistic and regional ties to Alsace.

Furthermore, analysis reveals a correlation between the perception of regional ties to Alsace and language choice in trans-border communication: Persons who construct a shared regional cohesiveness across the border tend to use the Alemannic dialect when interacting with Alsatians, whereas persons who do not perceive such a cohesiveness mainly use Standard German or French.
\end{abstract}

\section{$1 \quad$ Einleitung}

Das Oberrheingebiet wird traditionell als dialektale Einheit beschrieben. Die alemannischen Dialekte auf beiden Seiten des Rheins bilden eine Stufenlandschaft, in der sich Merkmale des Elsässischen auf der badischen Seite fortsetzen, zumeist etwas weiter rheinabwärts (cf. Ochs 1921, 1940). Die wichtigsten Isoglossen kreuzen also den Rhein und verlaufen nicht parallel zu ihm (cf. Maurer 1942: 281). Seit Ende des 20. Jahrhunderts wird diese sprachliche Einheit aber in Frage gestellt (cf. Klausmann 1990). Jüngere Arbeiten belegen eine zunehmende sprachliche Divergenz der elsässischen und badischen Seite des Oberrheingebiets: Die Staatsgrenze wird, bedingt durch die Stabilität basisdialektaler Merkmale im linksrheinischen Alemannisch und deren zunehmendem Abbau auf rechtsrheinischer Seite, immer stärker zu einer Sprachgrenze

\footnotetext{
* Ich danke den beiden GutachterInnen für ihre hilfreichen Anmerkungen zum Text.
} 
(cf. Auer et al. 2017; Breuninger 2016). Gleichzeitig deutet sich an, dass bestimmte Teilregionen des badischen Oberrheingebiets, wie etwa der Kaiserstuhl (ein ehemaliges vulkanisches Gebiet nordwestlich von Freiburg), dieser Tendenz weniger stark folgen als andere (cf. Hansen-Morath 2016). Im Falle des Kaiserstuhls geht die stärkere dialektale Konservativität einher mit der Beobachtung, dass diese Region viele dialektale Eigenschaften mit dem Elsässischen teilt (cf. Ochs 1939). Gerade in diesem Gebiet haben eine Reihe von Merkmalen des Elsässischen ihren Weg über den Rhein gefunden und sind bis heute teilweise erhalten geblieben. Das Oberrheingebiet ist folglich als Dialektraum nicht allein dadurch uneinheitlich geworden, dass sich die badischen alemannischen Dialekte insgesamt immer stärker vom Elsässischen entfernen - die Advergenz der Dialekte einzelner badischer Teilregionen in Richtung des Standarddeutschen erfolgt mit unterschiedlichen Geschwindigkeiten (cf. Hansen-Morath 2016).

Angesichts dieser sprachlichen Entwicklungen wendet sich der vorliegende Beitrag der Frage zu, ob die Bewohner des badischen Oberrheingebiets eine gemeinsame regionale Identität mit dem Elsass teilen. Erste Beobachtungen deuten darauf hin, dass die Einstellungen der Badener - genau wie die der Elsässer - oft von negativen Stereotypen über die Bewohner der anderen Rheinseite und von national motivierten Abgrenzungen geprägt sind (cf. Pfeiffer/Auer 2019). Allerdings sehen manche Bewohner rheinnaher badischer Orte auch kulturelle und sprachliche Gemeinsamkeiten mit dem Elsass, die der traditionellen dialektologischen Perspektive auf das Oberrheingebiet entsprechen. Ziel des vorliegenden Beitrags ist eine Bestandsaufnahme der verschiedenen grenzüberschreitenden Identitätskonstruktionen im badischen Oberrheingebiet. Im Fokus der qualitativen Analysen steht die sprachliche und regionale Verbundenheit, die aus Sicht der Badener über den Rhein hinweg besteht. Der Begriff „regionale Verbundenheit“ ist im Folgenden sehr breit zu verstehen als Oberbegriff, der die kulturelle, soziale, politische, historische und charakterliche (auf die Mentalität bezogene) Ebene umfasst. Von Interesse ist zum einen, ob die links- und rechtsrheinischen Dialekte (noch) als ähnlich wahrgenommen und gegenseitig verstanden werden und ob die grenzübergreifende Kommunikation im Dialekt stattfindet, was zu Beginn des 20. Jahrhunderts wohl noch problemlos möglich war (cf. Maurer 1942: 284). Zum anderen wird untersucht, ob aus badischer Perspektive eine regionale Verbundenheit mit dem Elsass existiert, die etwa darin zum Ausdruck kommt, dass die Badener den Elsässern dieselbe Mentalität oder Kultur zuschreiben.

Die Analysen werden zeigen, dass je nach badischer Teilregion Unterschiede zwischen den grenzüberschreitenden Identitätskonstruktionen bestehen, die maßgeblich auf die Geschichte der einzelnen Regionen zurückgeführt werden können. Für den vorliegenden Beitrag stehen die unterschiedlichen politischen Verhältnisse in den einzelnen Regionen - insbesondere hinsichtlich der Beziehungen zum Elsass - bis zur Gründung des Großherzogtums Baden zu Beginn des 19. Jahrhunderts im Vordergrund, in dem das gesamte badische Oberrheingebiet aufging.

\section{Datenerhebung und Analysemethode}

Um die oben aufgeworfenen Fragen zu beantworten, wurden 127 leitfadengestützte soziolinguistische Interviews in einem qualitativen Analyseverfahren ausgewertet. Die Interviews wur- 
den in insgesamt 22 Erhebungsorten auf der badischen Seite des Oberrheins geführt (siehe Abbildung 1; ${ }^{1}$ zur Begründung der Zuordnung der Erhebungsorte zu den Regionen, siehe Abschnitt 3). An jedem dieser Orte wurden in der Regel acht Gewährspersonen interviewt, stratifiziert nach Alter (25-35 vs. 60-70), Geschlecht und beruflichem Milieu (handwerklich/landwirtschaftlich vs. kommunikationsorientiert, nach Mattheier 1994). Für manche Erhebungsorte waren aber nur zwei Interviews für die Forschungsfragen dieses Beitrags brauchbar (siehe unten). Die Erhebungen wurden von einer Exploratorin und einem Explorator aus dem alemannischen Sprachraum im Rahmen des Projekts FLARS² durchgeführt.

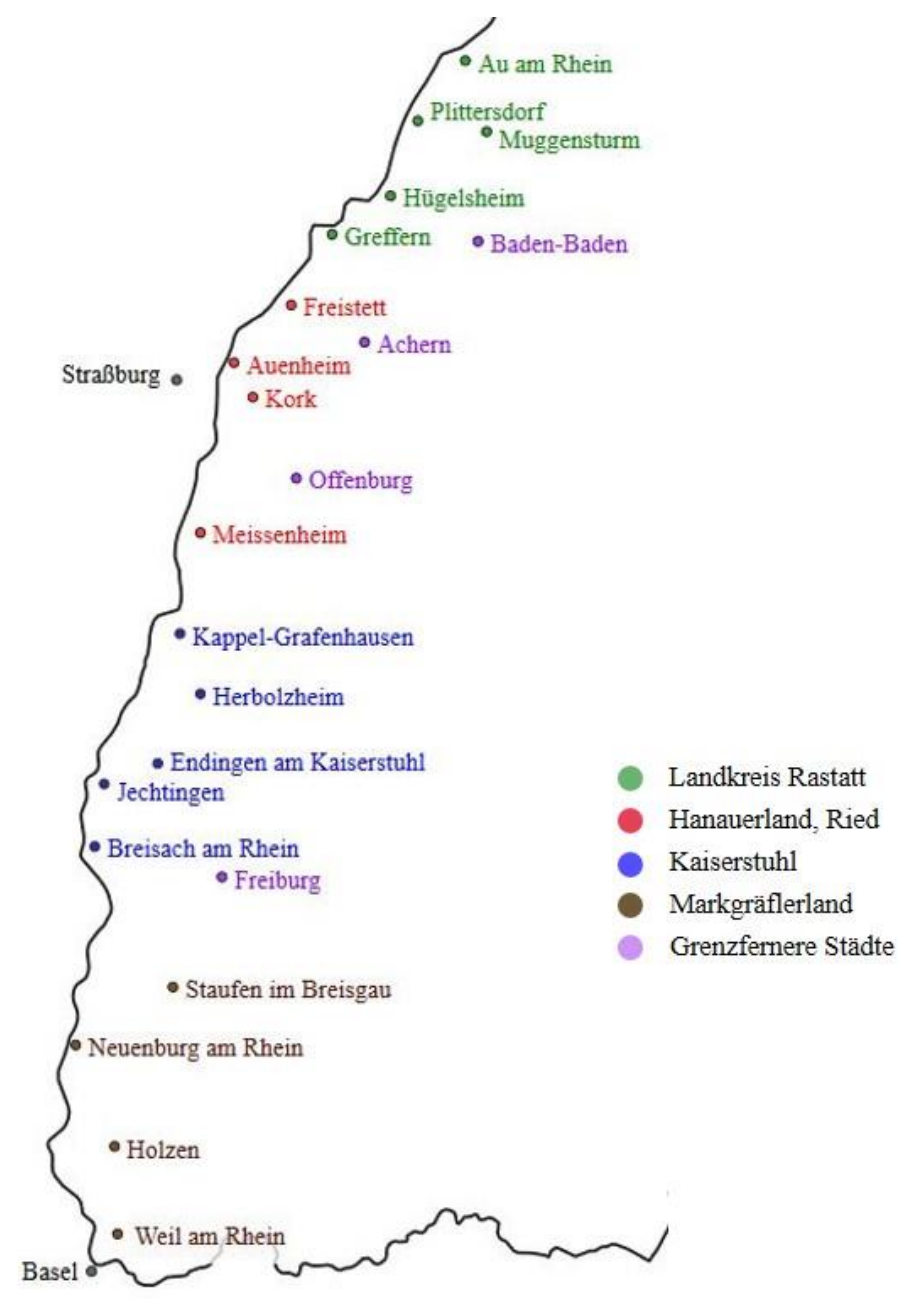

\section{Abbildung 1: Das Oberrheingebiet. Die 22 badischen Erhebungsorte nach Region. Zusätzlich sind Straßburg und Basel als Orientierungspunkte eingezeichnet.}

Für die Forschungsfragen dieses Beitrags sind die Aussagen der Interviewten zur Grenzregion und zu den Beziehungen zwischen den Badenern und den Elsässern von Bedeutung. Diese tauchen vor allem - aber nicht ausschließlich - im Kontext der folgenden Themenbereiche auf:

\footnotetext{
${ }^{1}$ Ich danke Andrea Streckenbach für ihre Unterstützung bei der Kartierung.

2 Das von der DFG und der ANR geförderte Kooperationsprojekt „Auswirkungen der Staatsgrenze auf die Sprachsituation im Oberrheingebiet“" (Frontière linguistique au Rhin Supérieur, FLARS) wurde von 2012 bis 2015 in Freiburg und Straßburg unter der Leitung von Peter Auer und Dominique Huck durchgeführt. MitarbeiterInnen waren Julia Breuninger, Pascale Erhart und Martin Pfeiffer. Für Einzelheiten zum Projekt und zur Datenerhebung, siehe Auer et al. (2015).
} 
- Haben Sie in Ihrem Alltagsleben das Gefühl, in einer Grenzregion zu leben?

- Fahren Sie oft ins Elsass? Aus welchen Gründen? Haben Sie dort Freunde oder Verwandte? Haben Sie beruflich Kontakt mit Elsässern?

- Welche kulturellen Gemeinsamkeiten oder Unterschiede gibt es diesseits und jenseits des Rheins?

- Haben die Leute auf der anderen Seite des Rheins einen anderen Charakter?

- Spricht man auf beiden Seiten des Rheins den gleichen Dialekt?

- Wie sprechen Sie im Elsass mit den Leuten?

Diese Themen wurden von den ExploratorInnen, entsprechend dem Verfahren des ,problemzentrierten Interviews“ (Witzel 1982, 2000), zu einem passenden Zeitpunkt in das Gespräch eingebracht (oder in manchen Fällen von den Gewährspersonen selbst angesprochen).

Die Auswertung beschränkte sich jedoch nicht auf Antworten auf ganz bestimmte Fragen, da die interessanten Themenbereiche nicht immer im Anschluss an die entsprechende Frage, sondern oft auch als unerwartete Antworten auf andere Fragen oder spontan im Diskurs auftauchten. Die Gespräche mit den InformantInnen wurden daher in der gesamten Länge ausgewertet, um möglichst alle relevanten Aussagen in die Analyse miteinbeziehen zu können. Für die Analysearbeit wurde für jedes Interview ein zusammenfassender Auswertungsbogen mit allen Gesprächsabschnitten erstellt, die mit der oben genannten Fragestellung in Verbindung standen. Die Auswertungsbogen dienten als Grundlage für die kontextsensitiven qualitativen Analysen, deren Ergebnisse auf demselben Bogen dokumentiert wurden.

Die Analysen zeigen, wie die grenzüberschreitenden (oder nationalen) Identitäten im Gespräch interaktiv hergestellt werden. Identitäten sind aus dieser Perspektive keine stabilen Entitäten, die ausschließlich in den Köpfen der Gewährspersonen als psychologische Gebilde oder außerhalb eines Gesprächs existieren, sondern ein Produkt der Interaktion zwischen InformantIn und InterviewerIn (cf. Bucholtz/Hall 2005). Identitäten werden in diesem Sinne dadurch konstruiert, dass sich die InformantInnen im Gespräch mit dem Explorator oder der Exploratorin auf eine bestimmte Weise positionieren (cf. Lucius-Hoene/Deppermann 2004). Die Analyse grenzüberschreitender Identitäten entspricht also aus gesprächsanalytischer Perspektive der Untersuchung interaktionaler Positionierungen zu den entsprechenden Themenbereichen, indem unter anderem der Inhalt des Gesagten, dessen Hervorbringung innerhalb des Gesprächskontexts und die Art und Weise der Versprachlichung detailliert in den Blick genommen werden. Im Zentrum der Analyse stehen diejenigen Identitätskonstruktionen, die sich in irgendeiner Weise etwa in sprachlicher, sozialer, kultureller, historischer oder politischer Hinsicht - auf das Oberrheingebiet als Grenzraum beziehen.

Über die Rekonstruktion individueller Identitäten hinaus geht der Beitrag der Frage nach, welche Identitätskonstruktionen über bestimmte soziale Gruppen hinweg geteilt werden, wie etwa eine ignorante und desinteressierte Haltung zu den Menschen auf der anderen Rheinseite (cf. Bister-Broosen 1998) oder überindividuell geteilte Vorstellungen, die die Mentalität oder die Sprache „der Anderen“ betreffen. Hier knüpfe ich an das aus der französischen Sozialpsychologie stammende Konzept der „représentations sociales“ an (cf. ausführlich zu diesem Konzept Jodelet 1993; Moscovici 2011; Abric 2016; im Folgenden übersetzt mit „,soziale Repräsentati- 
onen"), das in verschiedenen jüngeren Arbeiten als Instrument für die soziolinguistische Analyse fruchtbar gemacht worden ist (cf. Bothorel-Witz 2007; Bogatto et al. 2008; Auer 2018; Erhart 2019). Soziale Repräsentationen können als „Interpretationssysteme“ definiert werden, „die unsere Beziehung zur Welt und zu Anderen bestimmen“ und „das Sozialverhalten und die soziale Kommunikation ausrichten und organisieren“ (Jodelet 1993: 36, Übersetzung MP). Mit sozialen Repräsentationen sind die kollektiven Vorstellungen gemeint, die die Mitglieder einer sozialen Gruppe miteinander teilen (wie z. B. „Elsässer haben deutsche Tugenden“) und dazu nutzen können, der „Realität, mit der sie konfrontiert [sind], eine spezifische Bedeutung zu[zu]schreiben“" (Abric 2016: 17, Übersetzung MP). Es handelt sich bei solchen Repräsentationen um sozial erlernte Wissensbestände, ,die auch unabhängig von persönlichen Erfahrungen tradiert und von den Mitgliedern der Gemeinschaft gewusst, wenn auch nicht notwendigerweise verwendet oder gar geglaubt werden“ (Auer 2018: 7).

Wie die rekurrenten Zuschreibungen zeigen, die über die verschiedenen Interviews hinweg etwa bezüglich des Wesens oder der Sprache der Elsässer vorgenommen werden, spielen soziale Repräsentationen für die badischen Bewohner des Oberrheingebiets eine wichtige Rolle bei der Konstruktion grenzüberschreitender Identitäten. Diese gesellschaftlich vermittelten Wissensbestände sind nicht zuletzt auch deswegen von Bedeutung, weil grenzübergreifende Kommunikation im Oberrheingebiet faktisch nur noch sehr selten stattfindet, so dass eigene Erfahrungen als Basis für die Einschätzung „,der Elsässer“ und ihrer Sprache für viele Gewährspersonen keine tragfähige Ressource mehr darstellen. Die wenigen, meist oberflächlichen persönlichen Begegnungen zwischen Badenern und Elsässern sind im Allgemeinen auf wenige soziale Situationen wie das Einkaufen im anderen Land beschränkt (cf. Pfeiffer/Auer 2019).

\section{Grenzüberschreitende Identitäten im badischen Oberrheingebiet}

Hinsichtlich der grenzüberschreitenden Sprachwahl, der Einschätzung der eigenen Kompetenz, Elsässisch zu verstehen und der Frage nach einer regionalen grenzüberschreitenden Verbundenheit werden im Folgenden unterschiedliche Teilregionen miteinander verglichen, in die sich der badische Teil des Erhebungsgebiets gliedern lässt (siehe Abbildung 1). Diese Einteilung richtet sich nicht allein nach historischen und geografischen Kriterien, sondern zielt darauf $a b$, die ethnogeografischen Räume abzubilden, die in der Alltagswelt als Konstrukte existieren. Die Kriterien, die bei dieser Einteilung herangezogen wurden, werden im Folgenden näher erläutert.

Die Analyse beginnt mit der Region Hanauerland (3.1), die in historischer und politischer Hinsicht wohl am stärksten mit dem Elsass verbunden war. Zum badischen Hanauerland zählen die Erhebungsorte Freistett, Auenheim und Kork (Stadtteil von Kehl). Südlich schließt sich an das Hanauerland eine Gegend an, die von den Einheimischen als „Ried“ (,Sumpfgras, Schilf, Röhricht', cf. Pfeifer 2005: 1127) bezeichnet wird. In diesem Gebiet liegt der Erhebungsort Meißenheim. Er wird im Folgenden gemeinsam mit dem Hanauerland besprochen, weil dort sprachliche Gemeinsamkeiten mit dem Hanauerland vorliegen (cf. z. B. Auer/Breuninger/Pfeiffer 2017: $37 \mathrm{zu}$ den Sonderformen /bli/ und /blin/ für bleiben und ibd. 39f. zur Palatalisierung von mhd. $\hat{u}$, die dort nicht auftritt) und sowohl den Bewohnern des Hanauerlands als auch des Rieds sprachliche und charakterliche Nähe zu den Elsässern zugeschrieben wird (cf. die Aussagen der Stadtbewohner in 3.4). Anschließend richten wir den Blick auf den Kaiserstuhl 
(3.2), der sich traditionell durch eine besondere sprachliche Nähe zum Elsass auszeichnet. Neben den Orten Endingen und Jechtingen im Norden und Breisach am Südwestrand des Kaiserstuhls werden in diesem Abschnitt auch Orte aus der Umgebung einbezogen, die nicht zum Kaiserstuhl im engeren Sinne zählen: Herbolzheim und Kappel-Grafenhausen im Norden des Kaiserstuhls. Diese Einteilung erscheint dadurch gerechtfertigt, dass die traditionellen Dialekte dieser Orte sich in phonologischer Hinsicht ähnlich verhalten wie die Kaiserstühler Dialekte (cf. etwa Auer/Breuninger/Pfeiffer 2017: 39f. zur Palatalisierung von mhd. $\hat{u}$ ) und dass sich zwei Bewohner dieser Orte selbst der Region Kaiserstuhl zuordnen. Es folgen der Landkreis Rastatt am nördlichen Oberrhein (3.3), die grenzferneren Städte (3.4) und das Markgräflerland am südlichen Oberrhein (3.5), zu dem im vorliegenden Beitrag die Erhebungsorte Weil, Holzen, Neuenburg und Staufen gezählt werden. Während Weil und Holzen Teil des für das Markgräflerland namensstiftenden historischen Territoriums der „Oberen Markgrafschaft“ (Hoffmann 1994: 224) waren, lagen Neuenburg und Staufen immer außerhalb dieses Gebiets (Staufen nördlich, Neuenburg westlich). Beide Orte gelten heute aus Sicht vieler Bewohner des Oberrheingebiets dennoch als Teil der „Region Markgräflerland“, die in diesem Verständnis von der Schweizer Grenze bis zum Ende des „Reblands“ bei St. Georgen südlich von Freiburg reicht, also im Vergleich zur Oberen Markgrafschaft ein deutlich größeres, nach Westen und Norden ausgedehntes Gebiet umfasst (cf. Hoffmann 1994: 224f.). Für eine Festlegung der Region Markgräflerland in diesem ethnogeografischen Sinne spricht auch, dass eine der Gewährspersonen aus den betreffenden Orten (Neuenburg 1) ihren Wohnort explizit dem Markgräflerland zuordnet und eine andere Gewährsperson (Weil 2) sogar auch Basel hinzuzählt (siehe 3.5). Die abschließende Diskussion reflektiert die Faktoren, von denen die grenzüberschreitenden Identitätskonstruktionen abhängen, und zeigt Perspektiven für weiterführende Forschungen auf (Abschnitt 4).

In den südlichen Erhebungsorten einschließlich des Kaiserstuhls bis zur Schweizer Grenze gibt es jeweils nur zwei Gewährspersonen (einen Mann und eine Frau aus der jüngeren Gruppe mit handwerklich-landwirtschaftlichem Beruf), die sich ausführlich zum Elsass äußern. Dies ist darauf zurückzuführen, dass nur diese beiden Interviews im Rahmen des Projekts FLARS, die restlichen sechs aber im Vorgängerprojekt $\mathrm{REDI}^{3}$ mit etwas anderen inhaltlichen Schwerpunkten durchgeführt wurden. Betroffen sind die Erhebungsorte Herbolzheim, Endingen am Kaiserstuhl, Breisach am Rhein, Freiburg im Breisgau, Staufen im Breisgau, Neuenburg am Rhein, Holzen und Weil am Rhein; einzige Ausnahme ist der Erhebungsort Jechtingen am Kaiserstuhl, in dem alle acht Interviews im Rahmen des Projekts FLARS durchgeführt wurden. Hinsichtlich der Fragestellung des vorliegenden Beitrags ergibt sich damit für den Kaiserstuhl (Herbolzheim, Endingen, Breisach), die grenzferneren Städte (Freiburg) und insbesondere für das Markgräflerland (Staufen, Neuenburg, Holzen und Weil) eine reduzierte Datenlage. In letzterer Region lagen ausschließlich Interviews mit jüngeren InformantInnen zur Auswertung vor. Dieser Punkt muss im Folgenden mitreflektiert werden. An den entsprechenden Stellen wird im Text darauf verwiesen.

\footnotetext{
${ }^{3}$ Im Projekt „Regionaldialekte im alemannischen Dreiländereck“, das unter der Leitung von Peter Auer durchgeführt wurde, sind die Dissertationen von Sandra Hansen-Morath (2016) und Philipp Stoeckle (2014) entstanden.
} 


\subsection{Hanauerland}

Das Hanauerland, unter dem heutzutage der Großraum Kehl (etwa zwischen Hohnhurst und Lichtenau) verstanden wird, steht historisch in einer besonders engen Beziehung zum Elsass. Im 13. Jahrhundert fielen etwa 115 links und rechts des Rheins gelegene Ortschaften an die Herrschaft von Lichtenberg aus dem Unterelsass (cf. Fluck 1974: 24). Als Graf Philipp I. von Hanau im Jahre 1458 eine Tochter des letzten Lichtenbergers heiratete, trat für dieses Herrschaftsgebiet der Name Hanau hinzu (cf. ibd.). Das Territorium der Grafschaft blieb in derselben Größe über 500 Jahre hinweg (linksrheinisch bis 1793, rechtsrheinisch bis 1803) erhalten (cf. Rusch 1940: 200). Rusch (1940: 205) stellt daher fest, dass „Sprache, Sitten, Gebräuche, Namen und Einrichtungen jahrhundertelang auf beiden Seiten des Rheins im Hanauerlande die gleichen geblieben“ seien. ${ }^{4}$ Die Bezeichnung „Hanauerland“ wird von der rechtsrheinischen Bevölkerung bis heute verwendet. In sprachlicher Hinsicht hat das Hanauerland viele Neuerungen aufgegriffen, die vom nahegelegenen Straßburg ausgingen und über Kehl auf die rechte Rheinseite gelangten (cf. Schrambke 2012: 137).

Wie der folgende Interviewausschnitt zeigt, sehen die Bewohner des Hanauerlands (und des Rieds) enge Verbindungen zwischen ihrer Region und dem Elsass - sowohl sprachlich als auch hinsichtlich der Mentalität und Kultur (in allen folgenden Transkripten werden die Sprecherkürzel „G“ für Gewährsperson und „E“ für ExploratorIn verwendet):5

(1) GP_1391, 50:40, 60-70 J.

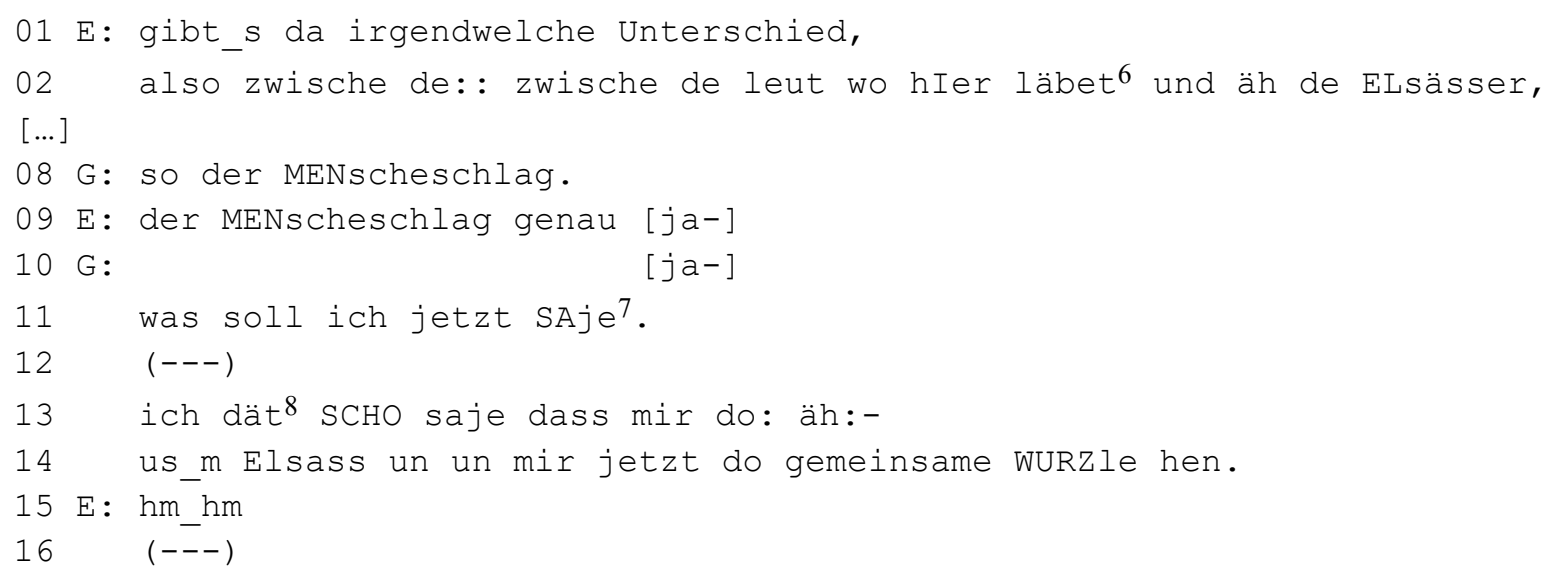

\footnotetext{
${ }^{4}$ Solche Aussagen aus einem Sammelband mit dem Titel Das Elsass, der während des Zweiten Weltkriegs publiziert wurde, sind mit aller Vorsicht zu betrachten, da sie den Anspruch des nationalsozialistischen Regimes auf die linksrheinischen Gebiete untermauerten. Selbst wenn man unterstellt, dass die Herausgabe dieses Buches in erster Linie politische Zwecke verfolgte (was unter anderem der pathetische Stil verschiedener Beiträge nahelegt, cf. etwa Rusch 1940: 205, „Urdeutsch ist das Land links des Rheines geblieben - urdeutsch wird es bleiben!“), bleibt dennoch für den vorliegenden Beitrag der interessante Anhaltspunkt bestehen, dass für Ruschs Beitrag gerade das Hanauerland als Paradebeispiel für elsässisch-badische Verbundenheit ausgewählt wurde.

${ }^{5}$ Neben der Beispielnummerierung sind Identifikationsnummer der Gewährsperson, Beginn des Gesprächsausschnitts und Zugehörigkeit zur jeweiligen Altersgruppe angegeben. Die Transkripte folgen den Konventionen von GAT 2 (Selting et al. 2009). In diesem und einigen folgenden Ausschnitten sind Nebensequenzen, die nicht von Interesse sind, ausgelassen und im Transkript durch ,[...]“ markiert.

${ }^{6}$ Standarddeutsche Form: leben.

${ }^{7}$ Standarddeutsche Form: sagen.

${ }^{8}$ Standarddeutsche Form: täte/würde.
} 


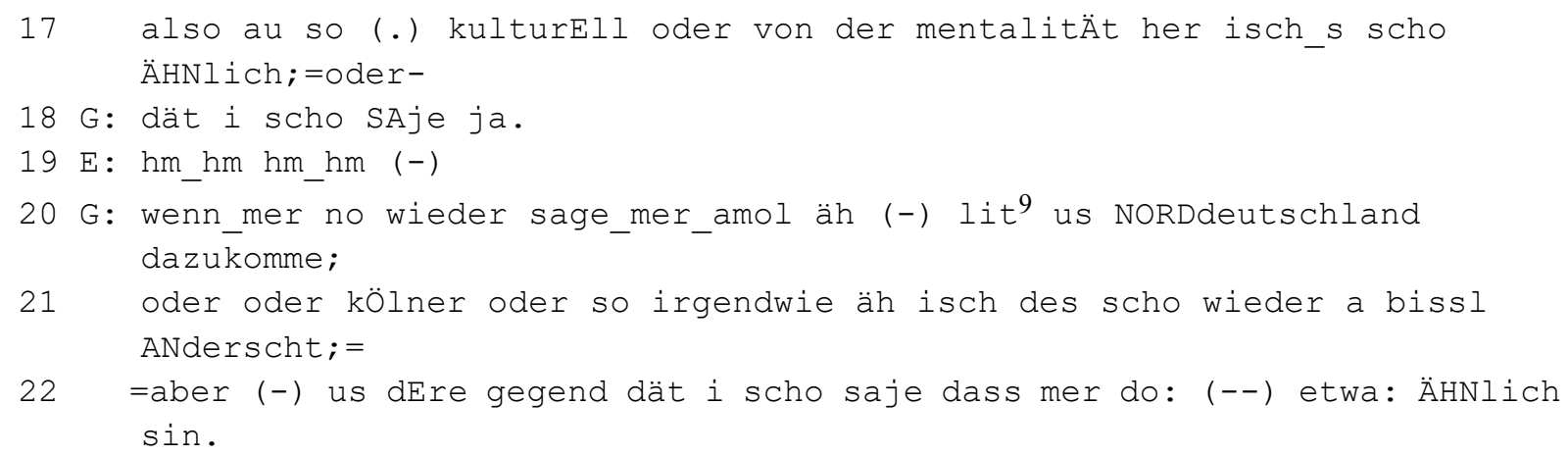

Auf die Frage nach Unterschieden in der Wesensart zwischen Badenern und Elsässern stellt der Informant eine Kollektivität her, die Badener und Elsässer als Teil desselben „Menschenschlags" umfasst, weil sie ein historisches Erbe teilen (siehe die Metapher gemeinsame WURZle, Z. 14). Zur Verdeutlichung dieser Verbundenheit stellt er einen Kontrast her zwischen einem grenzübergreifenden „Wir“ (mir do, Z. 13) und einer Alterität, die Personen aus anderen Gegenden Deutschlands umfasst (lit us NORDdeutschland, kÖlner, Z. 20/21). Er konstruiert also eine regionale Identität, für die die Staatsgrenze keine Rolle zu spielen scheint: Die Unterschiede zwischen den Menschen innerhalb Deutschlands sind für ihn größer als zwischen den elsässischen und badischen Bewohnern des Oberrheingebiets.

Eine solche gemeinsame, mit den Elsässern geteilte Identität wird von den meisten Bewohnern des Hanauerlands gesehen. Diese Sichtweise steht häufig in Verbindung mit einem Bewusstsein für die frühere grenzüberschreitende politische Zusammengehörigkeit (Freistett $6^{10}$ erwähnt etwa, dass „,der größere Teil vom Hanauerland“ im Elsass liegt) und die daraus resultierenden Gemeinsamkeiten (Freistett 6 weist z. B. in kultureller Hinsicht auf die Ähnlichkeit der Trachten hin). Auch viele Gewährspersonen aus anderen Teilen des Erhebungsgebiets nehmen bei Bewohnern rheinnaher Dörfer des Hanauerlandes und des angrenzenden Elsass einen ähnlichen Charakter wahr (,die könnt ma grad verwurschtle, des passt“", Achern 7).

Nicht nur auf der Ebene der Mentalität, sondern auch hinsichtlich der Sprache sehen die Gewährspersonen aus dem Hanauerland enge Verbindungen mit dem Elsass. Diese Sichtweise kann durch die Nennung konkreter geteilter sprachlicher Merkmale zum Ausdruck kommen, die den InformantInnen bewusst sind (z. B. das „Ihrzen“, also die Verwendung der 2. Person Plural als formelle Anredeform, cf. Kork 8, das als typisch Elsässisch gilt und in keiner der anderen Regionen als verbindendes Merkmal genannt wird), oder aber in allgemeinen Beurteilungen, wie im folgenden Ausschnitt:

(2) GP_1391, 7:53, 60-70 J.

01 E: aber wenn die jetz äh in ihrem ELsässisch schwätze-

02 dann [verstehn sie des ganz norMAL-=oder-]

$03 \mathrm{G}$ : [des isch ungef des isch desSELbe; ]

04 des isch desselbe;

\footnotetext{
${ }^{9}$ Standarddeutsche Form: Leute.

${ }^{10}$ Durch die Nennung von Ortsname und Zahl, wie hier „Freistett 6“, wird auf eine aus diesem Erhebungsort stammende Gewährsperson verwiesen, die aus Platzgründen nur im Fließtext (ohne ausführliches Transkript) zitiert werden kann. Diese Zitate, bei denen der Inhalt im Fokus steht, wurden größtenteils ins Standarddeutsche übersetzt.
} 
im gegenteil des isch noch a bissl (.) BREIter so;

noch a weng Usgeprägter s ELsässisch;

Dieser Ausschnitt zeigt, wie nahe sich aus Sicht des Informanten sein eigener Dialekt und das Elsässische stehen. Er wartet zum einen nicht das Ende der Frage ab, ob er das Elsässische ganz norMAL (Z. 02) verstehe, und antwortet zum anderen nicht dem Format der assertiven Frage entsprechend explizit bejahend oder verneinend. Vielmehr gibt er mit des isch desSELbe (Z. 03) in Überlappung mit der Exploratorin eine nur implizit bejahende Antwort und behandelt damit die Frage (obwohl sie in epistemischer Hinsicht als vorsichtig formuliert gelten kann, weil die enthaltene Assertion dem Informanten bereits ein Verständnis des Elsässischen zuschreibt) als nicht adäquat: Die Exploratorin stellt etwas in Frage (sein Verständnis des Elsässischen), was für den Informanten selbstverständlich gegeben ist. Anschließend schränkt er seine Antwort allerdings geringfügig ein, indem er auf den etwas breiteren und ausgeprägteren Charakter des Elsässischen hinweist (Z. 05/06).

Alle Gewährspersonen aus dem Hanauerland (nur eine äußert sich dazu nicht) geben zu verstehen, dass ihnen das Elsässische keine Verständnisprobleme bereitet. Sie weisen aber teilweise darauf hin, dass Bewohner anderer badischer Regionen Verständnisprobleme mit dem Elsässischen hätten.

Fast alle, auch die meisten Gewährspersonen aus der jüngeren Gruppe, geben zudem an, dass sie im Kontakt mit Elsässern zumindest in manchen Situationen den Dialekt als Kommunikationsmittel wählen. Teilweise weisen sie jedoch darauf hin, dass die Möglichkeiten des grenzüberschreitenden Dialektgebrauchs eingeschränkt sind. Während aus ihrer Sicht in elsässischen Dörfern und im Kontakt mit älteren Elsässern die Wahl des Dialekts in der Regel funktioniert, ist in Straßburg und mit jüngeren Elsässern die Kommunikation im Dialekt nur selten möglich, weil diese häufig kein Elsässisch (mehr) sprechen (z. B. Auenheim 3).

Die sprachliche Verbundenheit des Hanauerlands mit dem Elsass nehmen auch viele Gewährspersonen aus anderen Teilen des Oberrheingebiets wahr. Viele InformantInnen aus dem nördlich angrenzenden Landkreis Rastatt (Greffern 1, 2, 6, Hügelsheim 4, 6, 8, Plittersdorf 4) und grenzferneren Städten (Offenburg 7, Achern 5, 7, 8) schreiben dem Dialekt im Hanauerland eine größere Nähe zum Elsässischen zu als dem eigenen. Zudem zeichnet sich dieser Dialekt in der Fremdwahrnehmung durch ein Alleinstellungsmerkmal aus: Nur im Hanauerland finden sich Berichte darüber, dass eine der Gewährspersonen (Auenheim 6) bzw. Personen aus umliegenden Dörfern des Hanauerlands (Kork 2) anhand ihres Dialekts entweder von anderen Badenern oder sogar von Elsässern für Elsässer gehalten wurden.

Das Hanauerland ist also in den Augen seiner Bewohner und auch aus Sicht der umliegenden badischen Regionen sehr eng mit dem Elsass verbunden - sowohl hinsichtlich der Mentalität als auch hinsichtlich der Sprache.

\subsection{Kaiserstuhl}

Der Kaiserstuhl ist als der „salienteste ethnodialektale Raum“ (Stoeckle 2014: 509) des alemannischen Dreiländerecks wohlbekannt. Dem Kaiserstuhl wird starke Dialektalität zugeschrieben (cf. ibd.) und er gilt traditionell als eine Region, die dem Elsass nahesteht. Dies mag 
unter anderem mit der großen Bedeutung des Weinbaus für den Kaiserstuhl und die angrenzende elsässische Region zusammenhängen, auf die in den Interviews immer wieder hingewiesen wird. So ist auch der Volkskundler Busse (1939: 254) der Meinung, dass der Kaiserstühler zwar zum Breisgau gehöre, ,,aber seiner Sprache und seinem Wesen nach geht er eher mit dem Elsässer denn mit dem Schwarzwälder“. Gerade hinsichtlich der Sprache wird oft darauf hingewiesen, dass der Kaiserstuhl Innovationen aus dem Elsass aufgenommen habe, die insbesondere von der einflussreichen Freien Reichsstadt Straßburg ausgingen (cf. Noth 1993: 44). Ochs (1939: 171) geht sogar so weit zu behaupten: „Das Gesicht der ganzen Sprachlandschaft des Kaiserstuhls schaut nach Westen.“

Was die politische Geschichte angeht, so finden sich vor allem ab dem 11. bis ins 14. Jahrhundert Beziehungen zwischen dem Kaiserstuhl und dem Elsass sowie Lothringen. Das Geschlecht der Üsenberger herrschte in diesem Zeitraum über das Kaiserstuhlgebiet. Das Kernstück dieser Herrschaft bestand in den Vogtsrechten über die Gebiete des elsässischen Klosters Andlau (Dinghöfe mit den dazugehörigen Besitzungen in Endingen, Kiechlinsbergen und Bahlingen), die von den Üsenbergern vermutlich im 11. Jahrhundert erworben wurden; zudem hatten die Üsenberger das Dorf Ihringen als Lehen der Bischöfe von Metz inne (cf. Rest 1939: 91-94). Vom Hanauerland unterschied sich die Situation der Bevölkerung am Kaiserstuhl dadurch, dass das geschlossene Herrschaftsgebiet ausschließlich rechtsrheinisch angesiedelt war. Zudem hatten die Üsenberger Herrscher, die als Vögte Steuern bezogen und als Richter fungierten, ihren Sitz nicht links-, sondern rechtsrheinisch. Im 14. Jahrhundert zerfiel diese Herrschaft, so dass ein Teil des Kaiserstuhlgebiets an die Markgrafschaft Hachberg und ein anderer an Österreich fiel. Diese Verhältnisse blieben bis zum Beginn des 19. Jahrhunderts bestehen. Maurer (1942: 284) weist darauf hin, dass der Breisgau und der Sundgau (Elsass) durch die Zugehörigkeit zu Vorderösterreich „,durch die Jahrhunderte in enger Beziehung“ gestanden haben. Dieser Hinweis ist sicherlich berechtigt, jedoch handelte es sich nicht um ein geschlossenes Herrschaftsgebiet, das mit dem Hanauerland vergleichbar wäre. Nur an wenigen Stellen bestand eine territoriale Verbindung dieser vorderösterreichischen Gebiete über den Rhein hinweg.

Eine gemeinsame grenzüberschreitende Identität wird auch im Kaiserstuhlgebiet von vielen Gewährspersonen wahrgenommen, wie die folgenden Aussagen eines jüngeren Informanten zeigen. Er sieht, wie der ältere Informant aus dem Hanauerland, eine Verbundenheit mit den Elsässern und bezieht sich auf den häufig anzutreffenden Topos des Elsässers, der „,zwischen den Deutschen und den Franzosen“ steht. Seiner Meinung nach gibt es „zwei verschiedene Menschenschläge“ im Elsass, nämlich „Franzosen“ und „Elsässer“, wobei „der Elsässer mehr Deutscher ist als Franzose“, weshalb man mit ihm besser zusammenarbeiten könne:

(3) GP_1461, 24:56, 25-35 J.

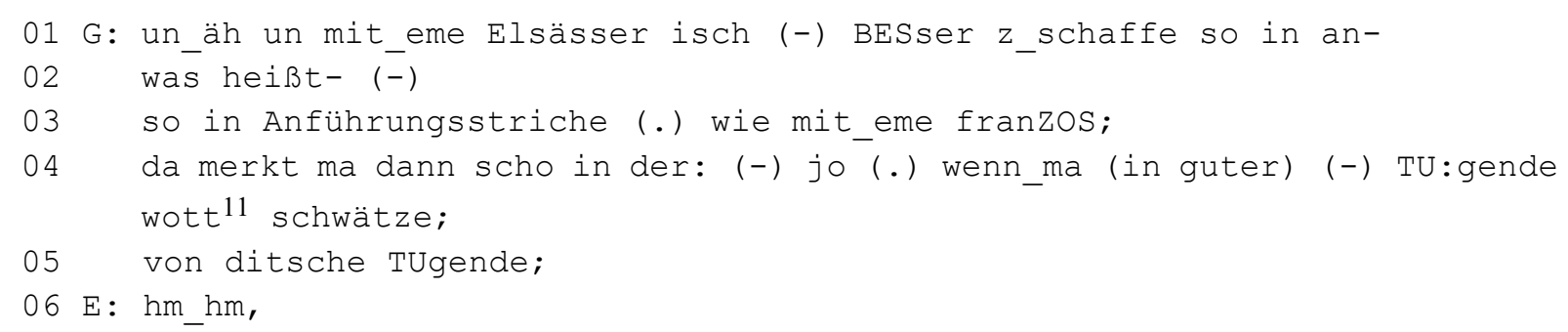

\footnotetext{
${ }^{11}$ Standarddeutsche Form: wollte.
} 


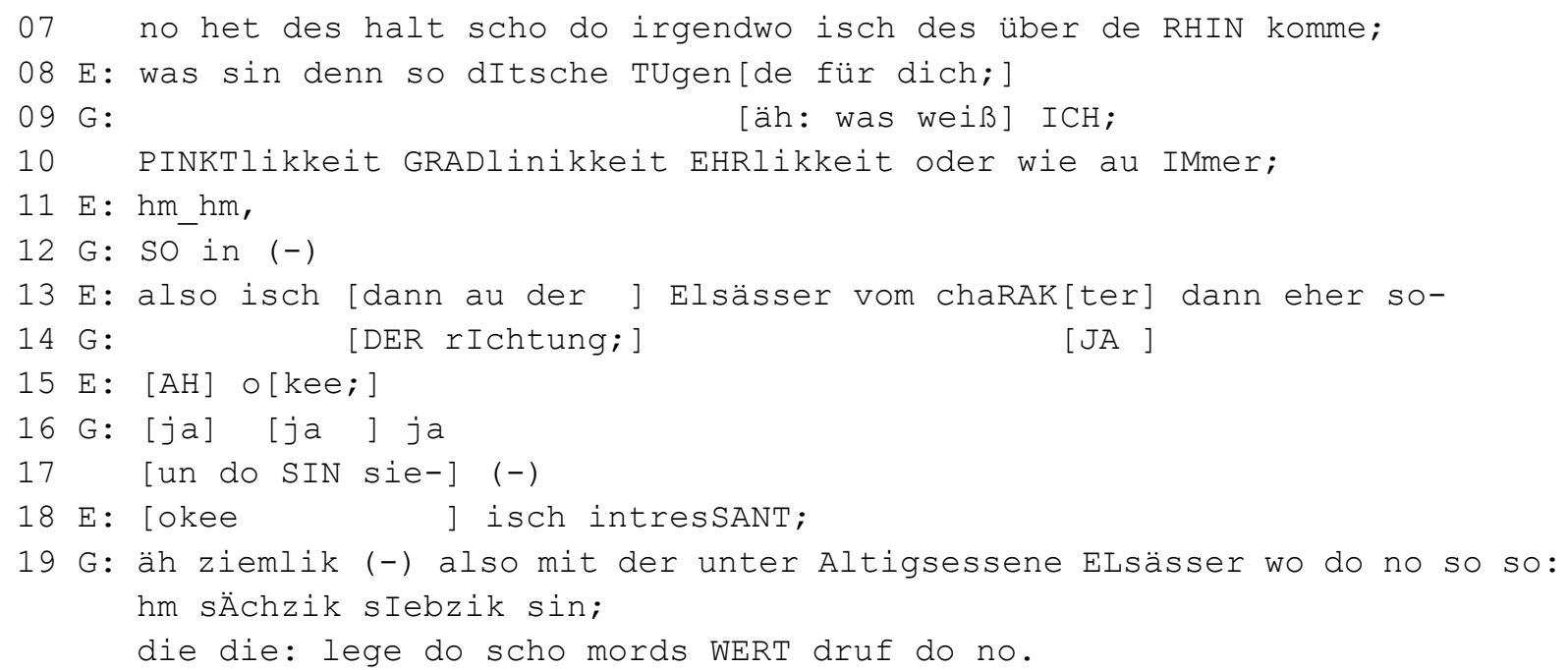

Dieser Informant konstruiert eine Nähe zwischen „dem Deutschen“ und „dem Elsässer2 und grenzt gleichzeitig beide von „dem Franzosen“ ab. Anders als in (1) sieht diese Gewährsperson keine durch regionale Zugehörigkeit zum Oberrheingebiet begründete Verbindung zwischen dem Elsass und Baden, sondern eine Verbindung zwischen dem Elsass und Deutschland: Als verbindende Eigenschaften schreibt er den Elsässern dItsche TUgende (Z.05) zu, also charakterliche Merkmale „des Deutschen“, die nicht nur für Badener gelten. Außerdem weist er durch die Bewegungsmetapher über de RHIN komme (Z. 07) explizit darauf hin, dass er den Ursprung dieser Tugenden in Deutschland sieht und von einer (vermutlich durch die gemeinsame Geschichte bedingte) Übernahme durch die Elsässer ausgeht, die sich auch heute noch im Charakter alteingesessener Elsässer im Alter zwischen sechzig und siebzig niederschlägt (Z. 19). Hier zeigt sich eine im gesamten badischen Oberrheingebiet anzutreffende Tendenz, eine gemeinsame deutsch-elsässische Mentalität - wenn sie denn überhaupt wahrgenommen wird - tendenziell in Bezug auf die ältere Generation von Elsässern zu konstruieren.

Diese Gemeinsamkeiten sind jedoch in den Augen vieler InformantInnen fragil und im Rückgang begriffen, wobei in den meisten Fällen die politischen Verhältnisse auf der elsässischen Seite als ausschlaggebend für die divergente Entwicklung angesehen werden. Die badischen Gewährspersonen nehmen einen starken Einfluss der Zugehörigkeit zum französischen Nationalstaat auf die Identität der Elsässer wahr (z. B. Kappel 7: „die Jungen werden jetzt alle ${ }^{2}$ sin jetzt alle französisch ne“, ,,der Franzose hat sowieso Nationalstolz und so langsam s: die haben halt, sie sagen auch wir sind jetzt in Frankreich eingegliedert, wir ${ }^{?}$ die müssen sich mit dem abfinden“; Jechtingen 4: ,ja gut das sind halt jetzt Franzosen geworden"). ${ }^{12}$ Dennoch zeigt sich gerade darin, dass die Gewährspersonen eine zunehmende Divergenz wahrnehmen und diese teilweise auch bedauern, dass sie von früheren engeren grenzübergreifenden Beziehungen ausgehen. Die meisten InformantInnen vom Kaiserstuhl und aus dem Hanauerland sehen im Gegensatz zu vielen Personen aus anderen badischen Regionen noch eine ursprüngliche Verbundenheit mit den Elsässern. Die Wahrnehmung wachsender Divergenz sollte also nicht allein als Konstruktion von Differenz zum Elsass gesehen werden, sondern auch als ein Beleg für die Wahrnehmung grenzüberschreitender Verbundenheit.

12 Das Symbol '?’ markiert einen Abbruch der Äußerung durch Glottalverschluss. 
Die Aussagen von Gewährspersonen aus unterschiedlichen badischen Regionen lassen den Schluss zu, dass die traditionelle Sichtweise, der Kaiserstuhl stehe als Dialektregion dem Elsässischen nahe, auch heute noch verbreitet ist: Der Dialekt der Region Kaiserstuhl gilt im Vergleich zu anderen badischen Dialekten als besonders ausgeprägt und dem Elsässischen besonders ähnlich. Die Kaiserstühler beschreiben ihren eigenen Dialekt im Vergleich mit dem Elsässischen zwar ebenfalls oft als ähnlich, aber mit einer Reihe von Unterschieden. Elsässisch ist aus ihrer Sicht insgesamt ,stärker“ (Jechtingen 1) als der Kaiserstühler Dialekt. Zwar werden weder von den Kaiserstühlern selbst noch von Gewährspersonen aus anderen Regionen spontan sprachliche Merkmale genannt, die den Kaiserstuhl mit dem Elsass verbinden. Allerdings wird in den badischen Interviews immer wieder die $\hat{u}$-Palatalisierung, die auch in der Region Kaiserstuhl auftritt, als salientes Merkmal des Elsässischen genannt („die Elsässer haben doch so viel ,ü‘ drin“, Au am Rhein 3). Die dem Kaiserstuhl zugeschriebene sprachliche Nähe zum Elsass könnte also zumindest teilweise auf diese - wenn auch womöglich unbewusste Ähnlichkeit zurückzuführen sein.

Die allermeisten Gewährspersonen aus der Region Kaiserstuhl geben von sich an, das Elsässische zu verstehen und in der grenzüberschreitenden Kommunikation ihren Dialekt zu sprechen, wie der folgende jüngere Informant, der häufig als Handwerker im Elsass unterwegs ist (neben der Exploratorin ist außerdem noch ein zweiter Informant aus der älteren Gruppe (G2) anwesend):

(4) GP_1486, 14:44, 25-35 J.

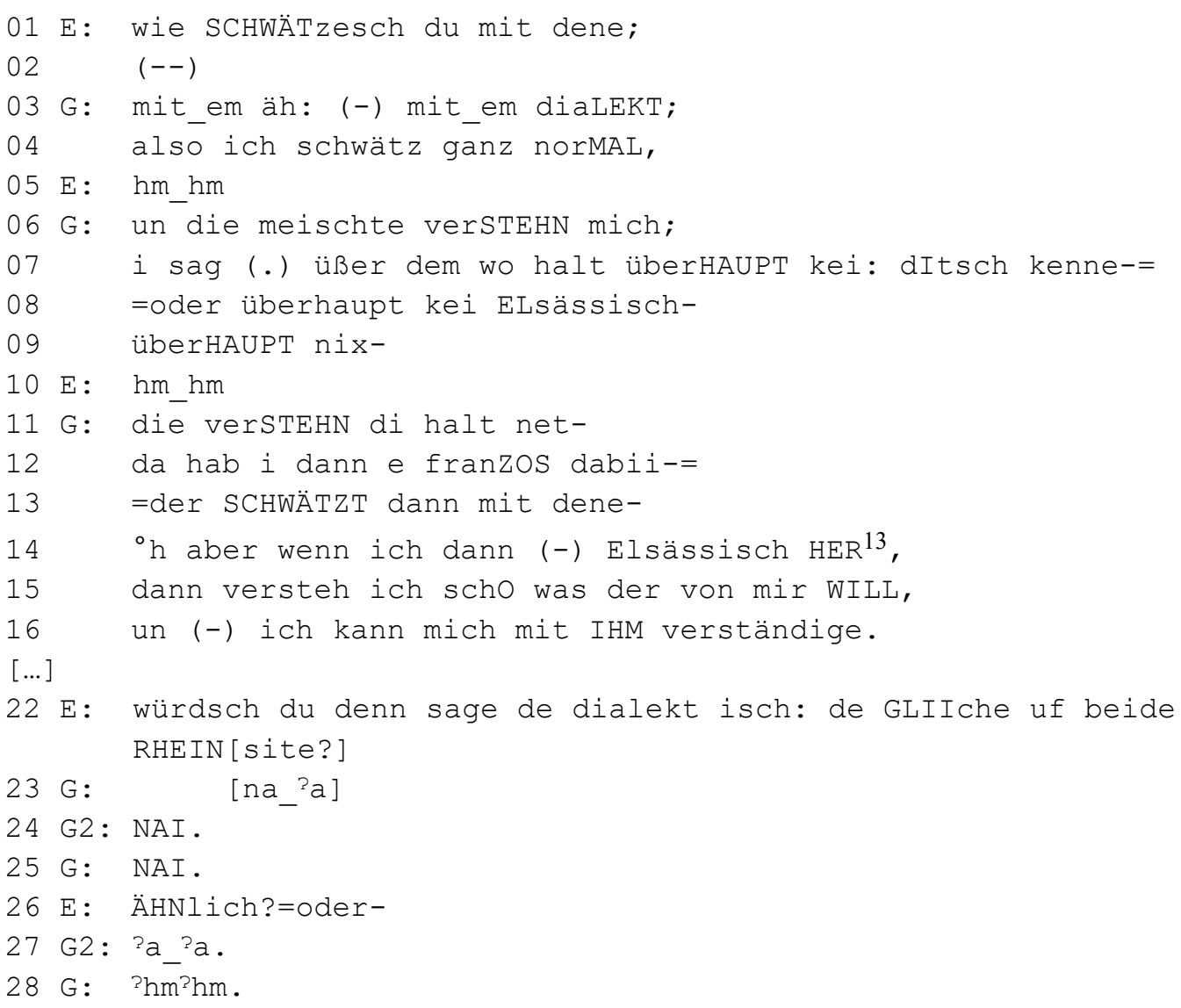

\footnotetext{
13 Standarddeutsche Form: höre.
} 


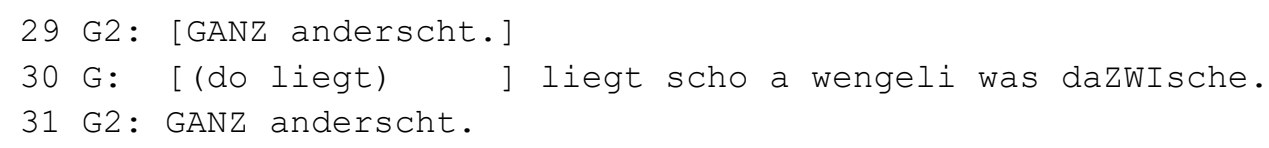

Der Informant beschreibt, dass die Verwendung des Alemannischen im Elsass in den meisten Fällen möglich ist (die meischte verSTEHN mich, Z. 06), jedoch nicht ohne Einschränkungen. Eine grundlegende Einschränkung besteht darin, dass er teilweise Leute antrifft, die überHAUPT kei: ditsch (Z. 07) oder überhaupt kei Elsässisch (Z. 08) können, so dass er mit ihnen überhaupt nicht in Kommunikation treten kann (die verSTEHN di halt net, Z. 11). Aber auch wenn die Gesprächspartner Elsässisch sprechen, scheint sich die grenzüberschreitende dialektale Kommunikation in ihrem Wesen von der Kommunikation unter Sprechern desselben Dialekts (i. e. des Dialekts einer relativ homogenen badischen Kleinregion) zu unterscheiden. Darauf deuten die Formulierungen des Informanten hin, die sein eigenes Verständnis des Elsässischen (versteh ich schO was der von mir WILL, Z. 15) bzw. das Verstandenwerden durch seinen elsässischen Kommunikationspartner (un (-) ich kann mich mit IHM verständige, Z. 16) beschreiben. Beide Formulierungen stellen den Aspekt des für praktische Belange ausreichenden Verständnisses in den Vordergrund (insbesondere in Z. 15 die Hervorhebung seiner Fähigkeit, die mit der elsässischen Äußerung verbundenen Implikationen für das eigene Handeln zu identifizieren), ohne dass ein Verständnis aller Details notwendigerweise gegeben sein müsste. In den meisten Fällen kann wohl nicht von einer Vertrautheit mit der Sprache des Anderen ausgegangen werden, wie sie in der Kommunikation unter Dialektsprechern desselben Orts (bzw. derselben elsässischen bzw. badischen dialektalen Kleinregion) gegeben ist. Das erscheint auch dadurch plausibel, dass sowohl die Gewährsperson (G) als auch eine weitere anwesende ortsansässige Person (G2) das Elsässische als dem Kaiserstühler Dialekt nicht ähnlich bzw. als GANZ anderscht beschreiben (Z. 22-31).

Bei der Frage, ob es sich auf beiden Rheinseiten um denselben Dialekt handelt, gehen die Meinungen der Gewährspersonen unabhängig von ihrem Herkunftsort oder Alter stark auseinander. Auch diejenigen Personen, die das Elsässische verstehen und im Elsass im Dialekt kommunizieren, kommen zu unterschiedlichen Urteilen, die von des isch desSELbe (siehe (2), Z. 03/04) bis zu GANZ anderscht (siehe (4), Z. 29/31) reichen. ${ }^{14}$ In vielen Fällen relativieren die Gewährspersonen aber ihre ursprüngliche Aussage und entwickeln eine ambivalente Beschreibung der links- und rechtsrheinischen alemannischen Dialekte als ähnlich, aber unterschiedlich (cf. Erhart 2019).

Genau wie der jüngere Informant in (4) sagen fast alle Gewährspersonen vom Kaiserstuhl (mit nur einer Ausnahme) über sich, dass sie Elsässisch problemlos verstehen. Die meisten InformantInnen verwenden ihren Dialekt auch im Gespräch mit Elsässern. Lediglich in der jüngeren Gruppe finden sich vereinzelt Personen, die im Elsass nicht auf ihren Dialekt zurückgreifen. Dies zeigt einerseits, dass der Dialekt als grenzüberschreitendes

\footnotetext{
${ }^{14}$ Vermutlich hängen diese Unterschiede mit dem Granularitätsgrad des Maßstabs zusammen, den die Gewährspersonen ihrem Vergleich zugrundelegen. Die Wahl eines groberen Maßstabs führt hier eher zur Einschätzung als gleich/ähnlich (Maßstab „dialektale Großräume“; eigener Dialekt und Elsässisch gehören zum Alemannischen) als die Wahl eines feineren Granularitätsgrads (Maßstab „dialektale Kleinräume“; die Dialekte der benachbarten badischen Regionen sind dem eigenen Dialekt ähnlicher als das Elsässische). Diese Überlegungen können an dieser Stelle nicht vertieft werden.
} 
Kommunikationsmittel in der Region Kaiserstuhl noch eine Rolle spielt, deutet aber andererseits darauf hin, dass die Bedeutung des Dialekts in dieser Funktion langsam abnimmt.

Wie im Hanauerland zeigt sich unter den Kaiserstühler Gewährspersonen eine recht enge Verbundenheit mit dem Elsass, insbesondere auf der sprachlichen Ebene. Eine gemeinsame grenzüberschreitende Identität scheint jedoch insgesamt etwas schwächer ausgeprägt zu sein.

\subsection{Landkreis Rastatt}

Die Erhebungsorte Greffern, Hügelsheim, Muggensturm, Plittersdorf und Au am Rhein im nördlich an das Hanauerland angrenzenden Landkreis Rastatt sind ebenfalls historisch mit dem Elsass verflochten. Immer wieder weisen die Gewährspersonen darauf hin, dass unter anderem Plittersdorf durch den vor der Rheinregulierung im 19. Jahrhundert ständig wechselnden Verlauf des Rheinstroms zeitweise linksrheinisch angesiedelt war (cf. auch Schrambke 1981: 44). Wie in anderen Teilen des Oberrheingebiets besaßen auch hier Badener wie Elsässer Ländereien auf der jeweils anderen Seite des Rheins, die im Zuge von Grenzbereinigungen teilweise getauscht wurden. Allerdings war die politische Verbundenheit mit dem Elsass in dieser Region, die länger als zwei Jahrhunderte Teil der Markgrafschaft Baden-Baden war, weniger stark als etwa im Hanauerland. Das Herrschaftszentrum der Markgrafschaft BadenBaden lag auf rechtsrheinischer Seite (erst im heutigen Baden-Baden, später in Rastatt) und nur ein flächenmäßig sehr kleiner und politisch wenig bedeutender Teil des Herrschaftsgebiets war linksrheinisch angesiedelt. In der Lebenswelt der Bewohner der Markgrafschaft bestand also wenig Anlass, sich nach Westen in Richtung Elsass auszurichten. Die Bevölkerung des kleinen rechtsrheinischen Gebiets des Hanauerlandes hingegen orientierte sich zwangsläufig in Richtung Elsass, wo der weitaus größere Teil des Hanauerlandes angesiedelt war, die Herrschaft residierte und insbesondere das nahegelegene Straßburg als wirtschaftliches, politisches und kulturelles Zentrum eine starke Anziehungskraft ausübte.

Im Landkreis Rastatt zeigt sich kein so einheitliches Bild wie im Hanauerland und am Kaiserstuhl. Einerseits gibt es eine Reihe von Gewährspersonen, auch in der jüngeren Gruppe, die eine Verbundenheit mit dem Elsass konstruieren, andererseits nimmt aber ein großer Anteil der InformantInnen deutliche Unterschiede wahr - sowohl auf der Ebene der Sprache als auch auf der Ebene der Mentalität.

Manche Gewährspersonen, wie die folgende jüngere Informantin, konstruieren eine regional bedingte Verbundenheit mit dem Elsass, die mit den Aussagen der Gewährspersonen im Hanauerland und am Kaiserstuhl vergleichbar ist:

(5) GP_1427, 11:56, 25-35 J.

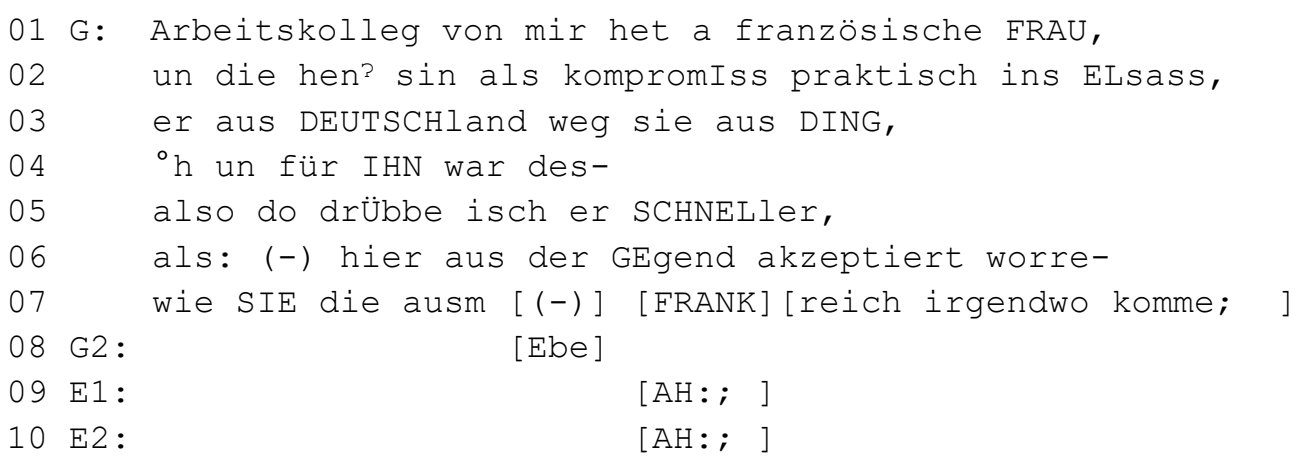


$11 \mathrm{G} 2$ :

[mir sind keine franzose] mir sin

ELsässer.

12 E2: SAge die;

13 G2: SAge die;

Diese Erzählung beginnt zu einem Zeitpunkt, als die beiden am Gespräch beteiligten Gewährspersonen bereits anhand mehrerer Beispiele (z. B. wechselnde linksrheinische und rechtsrheinische Ansiedlung des Wohnorts wegen unterschiedlicher Verläufe des Rheins; Bereitschaft der Elsässer, mit ihnen im Dialekt zu sprechen, aber nicht mit Badenern aus anderen Regionen) auf die historische und sprachliche „Verbundenheit“ zwischen Baden und dem Elsass hingewiesen haben. Schon die Platzierung der Erzählung im Gesprächsverlauf zeigt also an, dass sie weitere Evidenz für die Existenz einer grenzübergreifenden Verbundenheit liefern soll, was durch den Inhalt der Erzählung eingelöst wird. Das geschilderte Erlebnis ihres Arbeitskollegen legt nahe, dass Badener im Elsass willkommener sind als Franzosen (aus anderen Regionen als dem Elsass). Elsässer fühlten sich einem zugezogenen „Nachbarn“ aus der Oberrheinregion verbundener als einer Person aus einer anderen Region Frankreichs. Das Narrativ entwickelt - wie auch die Interpretation der Erzählung durch die zweite Gewährsperson zeigt (mir sin keine franzOse mir sin ELsässer, Z.11) - eine Fremdzuschreibung, die die regionale grenzüberschreitende Identität der Elsässer stärker gewichtet als deren nationale Identität als „Franzosen“.

Diese Sicht auf die Elsässer wird im Landkreis Rastatt allerdings von der Mehrheit der InformantInnen nicht geteilt. In dieser Region fällt auf, dass sich in der älteren Gruppe hinsichtlich der empfundenen Nähe zu den Elsässern kein so einheitliches Bild abzeichnet wie im Hanauerland oder am Kaiserstuhl. Einige Gewährspersonen, wie die folgende, sehen keine historisch, kulturell oder charakterlich begründete Verbundenheit, auf die sich die Konstruktion einer grenzüberschreitenden Identität stützen könnte. Dieser Informant spricht über das Verhältnis zu seinen elsässischen Arbeitskollegen, mit denen er wenig Kontakt hatte:

(6) GP_1403, 54:00, 60-70 J.

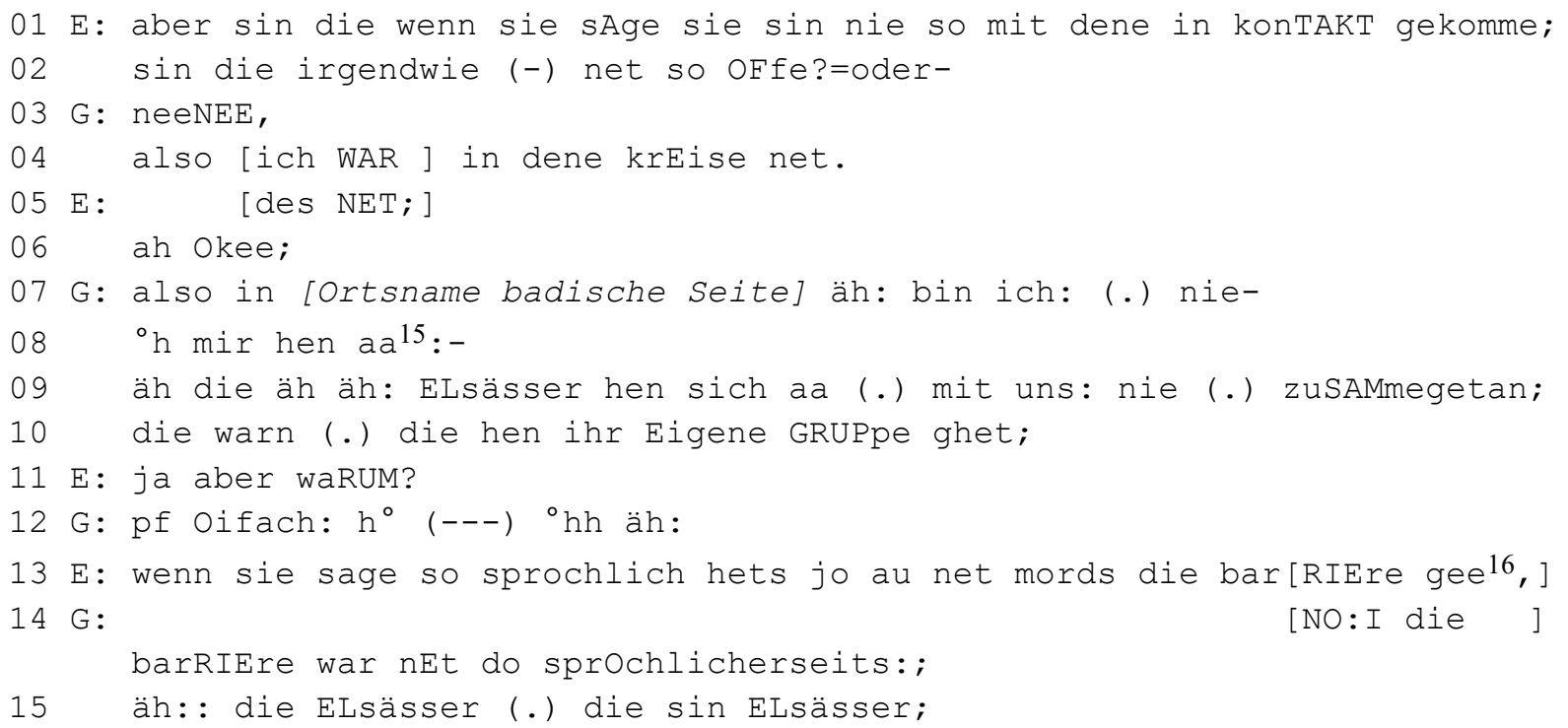

\footnotetext{
15 Standarddeutsche Form: auch.

16 Standarddeutsche Form: gegeben.
} 
$16 \mathrm{E}: \mathrm{hm} \_\mathrm{hm}<<$ lächelnd $>\mathrm{KKEE},>$

$17 \mathrm{G}$ : un mir warn (--) do hibbe ${ }^{17}$ für UNS .

$\mathrm{Zu}$ Beginn des Ausschnitts weist er die von der Exploratorin vorgeschlagene mögliche Begründung zurück, dass fehlende Offenheit der Elsässer der Grund für den fehlenden Kontakt gewesen sein könnte. Er weist stattdessen darauf hin, dass er nicht in denselben krEise (Z. 04) verkehrte wie die Elsässer, ohne diese Aussage zunächst weiter auszuführen. Für ihn scheint es selbstverständlich zu sein, dass „Elsässer“ und „Badener“ (uns, mir, Z. 09/17) jeweils eigene Gruppen bilden und wenig Kontakt zueinander haben. Wie die vielen Verzögerungsphänomene (Pausen, Dehnungen, Reparaturen und Neustarts, siehe Z. 07-10) zeigen, mit denen sein anschließender Erklärungsversuch verbunden ist, fällt es ihm schwer, Gründe für den fehlenden Kontakt zu den Elsässern anzuführen und zu versprachlichen. Seine Ausführungen machen aber deutlich, dass er in erster Linie die Elsässer für den fehlenden Kontakt verantwortlich macht (die äh äh: ELsässer hen sich aa (.) mit uns: nie (.) zuSAMmegetan, die hen ihr Eigene GRUPpe ghet, Z. 09/10). Er unterstellt ihnen ein Für-sich-bleiben-wollen, also gewissermaßen eine fehlende Initiative im Zugehen auf die Badener. ${ }^{18}$

Die Exploratorin fragt daraufhin explizit nach dem Grund für den fehlenden Kontakt zwischen den Gruppen (ja aber waRUM?, Z. 11). Sie gibt damit zu verstehen, dass das „Für-sichbleiben" für sie nicht selbstverständlich ist und der Informant aus ihrer Sicht bisher noch keine zufriedenstellende Erklärung gegeben hat - auch angesichts der Tatsache, dass er zu einem früheren Zeitpunkt auf die sprachlichen Gemeinsamkeiten zwischen dem Elsass und Baden hingewiesen habe (Z. 13). Der Informant signalisiert durch die Partikeln $p f^{19}$ und Oifach und das anschließende Ausatmen und Stocken (Z. 12), dass er keine befriedigende Begründung parat hat. Er bestätigt aber interessanterweise, dass es nicht an einer sprachlichen Barriere gelegen habe (Z. 14), schließt also zumindest die sprachliche Ebene als möglichen Grund für den fehlenden Kontakt aus. Schließlich gelingt es ihm, eine Erklärung anzudeuten: Er liefert die Antwort auf die warum-Frage in Form der Tautologie die ELsässer (.) die sin ELsässer (Z. 15). Damit erklärt er das Verbleiben der Elsässer innerhalb der eigenen Gruppe mit dem Wesen der Elsässer, schreibt diesem Kollektiv also die Eigenschaft zu, unter sich bleiben zu wollen, ohne irgendwelche weiteren Motivationen oder Gründe für die Existenz dieser Eigenschaft anzuführen. Durch diese tautologische Aussage markiert der Informant gleichzeitig den Endpunkt möglicher Erklärungen für diese Eigenschaft und zeigt der Exploratorin an, dass er die ihm durch die Frage auferlegte Begründungspflicht als erfüllt ansieht und weiteres Nachhaken zwecklos ist (siehe das lächelnde oKEE der Exploratorin in Z. 16 und das anschließende Ausbleiben weiterer Nachfragen).

\footnotetext{
17 Standarddeutsche Form: hüben.

${ }^{18}$ Der Grund, warum der Informant das Unterlassen des Aufeinanderzugehens nicht als beiderseitiges Versäumnis ansieht, sondern primär den Elsässern anlastet, bleibt unklar. Möglicherweise steckt dahinter die Erwartungshaltung, dass normalerweise die „Fremden“ auf die am Arbeitsort ansässigen Personen zugehen sollten und nicht umgekehrt.

${ }^{19}$ Baldauf-Quilliatre (2016) beschreibt für das Französische, dass die Verwendung von „pf“ in Gesprächen „disengagement“ anzeigen kann. Eine ähnliche Funktion erfüllt „pf“" auch in diesem Fall, nämlich das Anzeigen einer Loslösung von der Begründungspflicht, die die Exploratorin durch ihre Nachfrage aufbaut.
} 
Anders als die oben vorgestellten Gewährspersonen aus dem Hanauerland und dem Kaiserstuhl konstruiert dieser Informant aus dem Landkreis Rastatt keine Verbundenheit, sondern Differenz. Er zeichnet ein Bild von „den Elsässern“ und „den Badenern“ als separate Gruppen, die wenig Kontakt miteinander haben und unter sich bleiben, wofür er in erster Linie das Wesen der Elsässer verantwortlich macht.

Auch was die Beurteilung des Verhältnisses zum Elsass auf der sprachlichen Ebene angeht, ist die Gruppe von InformantInnen aus dem Landkreis Rastatt zwiegespalten. Einerseits kommt in manchen Aussagen über die Sprache dieselbe Verbundenheit zum Ausdruck, die aus der Sicht einiger InformantInnen aus dem Landkreis Rastatt zwischen Elsässern und den Bewohnern grenznaher badischer Dörfer besteht. Insbesondere in Plittersdorf, das eine Zeit lang linksrheinsch war, weisen mehrere InformantInnen darauf hin, dass die Elsässer ihren Dialekt erkennen und mit ihnen im Dialekt kommunizieren würden („Elsässer erkennen den Plittersdorfer Dialekt, die wissen, der kommt aus dem Grenzeck“, Plittersdorf 2), wohingegen sie bei anderen Badenern „komplett abblocken“ und nur Französisch sprechen würden. Andererseits sehen die meisten Gewährspersonen aus dem Landkreis Rastatt deutliche Unterschiede zwischen ihrem eigenen Dialekt und dem Elsässischen und weisen gleichzeitig auf große Ähnlichkeiten zwischen dem Elsässischen und dem Dialekt aus dem benachbarten Hanauerland hin (Antwort auf die Frage, ob das Elsässische dem eigenen Dialekt ähnlich sei: „eher wie im Hanauerland, eher wie Helmlingen, Freistett, hat mit unserem nicht so viel zu tun“, Greffern 2; ähnlich äußern sich Greffern 1, Greffern 6, Hügelsheim 4, Hügelsheim 6, Hügelsheim 8, Plittersdorf 4). Sie konstruieren also eine dialektale Nähe zwischen den Elsässern und den Bewohnern des Hanauerlands und grenzen sich gleichzeitig von beiden Gruppen ab.

Im Hanauerland und am Kaiserstuhl geben fast alle Gewährspersonen an, dass sie Elsässisch gut verstehen (einzige Ausnahme ist eine jüngere Informantin vom Kaiserstuhl). Dies trifft auch auf den Großteil der InformantInnen im Landkreis Rastatt zu, die auf eine gewisse Nähe ihres eigenen Dialekts zum Elsässischen hinweisen (,verstehe Elsässisch besser als weiter entfernte badische Dialekte, zum Beispiel aus Bühl“", Greffern 4). Allerdings berichten auch mehrere Personen von Verständnisproblemen (siehe den folgenden Ausschnitt (7)).

Anders als die Gewährspersonen im Hanauerland und am Kaiserstuhl greift im Landkreis Rastatt eine ganze Reihe jüngerer und älterer Gewährspersonen in der grenzübergreifenden Kommunikation nicht auf den Dialekt zurück, sondern auf das Französische (Hügelsheim 3), das „Hochdeutsche“ (Plittersdorf 6) oder einen „gemäßigten Dialekt“ (Hügelsheim 4, Muggensturm 3), wie auch der folgende ältere Informant. Auf die Frage, wie er denn mit den Leuten im Elsass spreche, antwortet er zunächst ,wir Badisch und die Elsässerisch und jeder versteht jeden“, relativiert diese Aussage dann aber bei der Nachfrage, ob es sich auf beiden Rheinseiten um denselben Dialekt handle:

(7) GP_1401, 34:30, 60-70 J.

$01 \mathrm{E}$ : isch des der: GLEIche dialekt eigentlich,

02 G: [NA:i ] [nai ]

$03 \mathrm{E}$ : [uf de] [uf de] oine un uf de andre seit vom RHEI,

$04 \mathrm{G}$ : äh: wenn mir: regelrecht BABBeln würde wie die au versteht kEiner de ANNer. 
$05 \mathrm{E}: \mathrm{JA}$ ?

06 G: ja ja do_isch nIx zu MAche;

07 ELsässerdÜtsch-

08 rIchtig Elsässer versteh Ich überHAUPT net.

$09 \mathrm{E}:$ AHja;

$10 \mathrm{G}$ : un dIe Uns dann aber AA net;

$11 \mathrm{E}:$ ah: [OKEE, ]

12 G: [un da] tu mer halt mol so bissl (.) net HocHdeutsch;

13 aber jeder soll sich AAstrenge dass_n $<<l a ̈ c h e l n d>$ de Anner

$14 \mathrm{E}$ :

[verSTEHT.]>

$[($ ( lacht $))]$

Der Informant ist der Meinung, dass grenzüberschreitende Kommunikation nur dann funktioniert, wenn Elsässer und Badener sich einander sprachlich anpassen. Ohne eine solche Akkomodationsleistung (jeder soll sich AAstrenge, Z. 13), die im Ergebnis immer noch zu einer mehr oder weniger dialektalen Varietät führt (siehe die Konzessivkonstruktion net Hochdeutsch aber..., Z. 12), sei gegenseitiges Verständnis nicht möglich (Z. 04). Er selbst verstehe richtigen elsässischen Dialekt überhaupt nicht (Z. 08), betont aber, dass die Elsässer dieselben Verständnisprobleme mit einem nicht abgeschwächten badischen Dialekt hätten (Z. 10). Diese Sicht steht im Kontrast zu den Aussagen der Gewährspersonen aus dem Hanauerland und vom Kaiserstuhl. Äußerungen, die auf die Notwendigkeit der Akkomodation in der grenzübergreifenden Kommunikation hinweisen, finden sich dort nicht. Die InformantInnen aus diesen Regionen geben in der Regel an, dass sie ganz norMAL mit den Elsässern sprechen (siehe (4), Z. 04), sich also gerade nicht AAstrenge müssen.

Neben der Herstellung eines sprachlichen Kontrasts zum Hanauerland (und dem Elsass) scheint in der Grenzregion bei Rastatt noch eine weitere Dimension für die Einordnung des eigenen Dialekts eine Rolle zu spielen. Häufig wird auf dialektale Unterschiede verwiesen, die die Entfernung zum Rhein als entscheidendes Kriterium heranziehen: „Je weiter weg vom Rhein, desto stärker nimmt das Verständnis ab“ (Greffern 7). Auch die umgekehrte Formulierung, dass eine größere Nähe zum Rhein mit besserem Verständnis des Elsässischen einhergehe, findet sich in den Daten (,Meine Mutter versteht noch Elsässisch, weil sie direkt am Rhein in [Ortsname] groß geworden ist", Muggensturm 4). Aus dieser Konstruktion dialektaler Differenzen, die auf Entfernungsunterschieden zum Rhein von wenigen Kilometern basiert, ergibt sich möglicherweise die Sonderrolle, die der etwas grenzfernere Ort Muggensturm innerhalb der Erhebungsorte im Landkreis Rastatt einnimmt. Hier weisen besonders viele Gewährspersonen auf Verständnisprobleme mit dem Elsässischen hin und geben an, mit Elsässern keinen Dialekt zu sprechen. Aus Muggensturm stammt auch die einzige Person unter den InformantInnen aus dem Landkreis Rastatt, die nach eigener Aussage das Elsässische nicht kennt („nur vom Hörensagen“, „ganz furchtbar“, Muggensturm 1) und deswegen keine Auskunft über die eigene Verständniskompetenz geben kann.

Im Landkreis Rastatt ergibt sich insbesondere im Vergleich zum Hanauerland, aber auch zum Kaiserstuhl, ein heterogeneres Bild: Einige InformantInnen nehmen zwar eine enge Verbundenheit mit dem Elsass wahr, aber für viele stehen die Unterschiede auf der anderen Seite des Rheins im Vordergrund. 


\subsection{Grenzfernere Städte}

Zum Erhebungsgebiet zählen auch die grenzferneren Städte Freiburg (Stadtteil Herdern), Offenburg, Achern (Stadtteil Oberachern) und Baden-Baden (Stadtteil Lichtental), die anders als etwa die grenznahen Städte Kehl (Stadtteil Kork, Hanauerland), Breisach (Kaiserstuhl) oder Neuenburg (Markgräflerland) keiner ethnogeografischen Region zugeordnet wurden und im Folgenden gemeinsam betrachtet werden. Über Jahrhunderte gehörten Freiburg (ab 1368) und Achern (ab 1551) zu Vorderösterreich (siehe 3.2). Offenburg war lange Zeit Freie Reichsstadt, Baden-Baden die Hauptstadt der gleichnamigen Markgrafschaft (siehe 3.3). Die etwas größere Entfernung dieser Städte zum Rhein (v. a. Achern) und deren Bedeutung als eigenständige Zentren (Freiburg, Offenburg, Baden-Baden) haben wohl dazu beigetragen, dass das Elsass seit jeher für viele der Bewohner kein Teil der alltäglichen Lebenswelt war. Zudem sind Städte im Allgemeinen stärker von Zuzug auswärtiger Personen betroffen, die mit ihrem sprachlichen Hintergrund und ihren andernorts geprägten Ansichten einen Einfluss auf die bestehenden Verhältnisse ausüben. Dies trifft jedoch auch auf die oben genannten Städte zu, die einzelnen Regionen zugeordnet wurden.

Die Stadtbewohner sehen deutlich weniger Gemeinsamkeiten zwischen sich selbst und den Elsässern, was die Mentalität oder Kultur angeht, als dies in den oben behandelten Regionen der Fall ist. Angesprochen auf die physisch nicht mehr vorhandene Grenze zwischen Deutschland und Frankreich, entwickelt die folgende jüngere Informantin eine Perspektive auf den Grenzraum, in der die politische Grenze zu Frankreich und ihre eigene Zugehörigkeit zum Nationalstaat Deutschland eine zentrale Rolle spielen. Auffällig sind in diesem Ausschnitt, wie in (6), die vielen Konstruktionsabbrüche, Selbstreparaturen, Pausen und Häsitationspartikeln. Die Verzögerungsphänomene in Z. 11-19 deuten nicht, wie die relativ zügige Antwort auf alle FÄLle (Z. 04) zeigt, auf fehlendes Wissen oder Probleme bei der Festlegung auf eine bejahende oder verneinende Antwort hin. Vielmehr können sie - da Verzögerungsphänomene in einer solchen Anhäufung bei dieser Gewährsperson ansonsten nicht zu beobachten sind - als Anzeichen dafür interpretiert werden, dass die Informantin die Existenz einer französischdeutschen Grenze und der damit verbundenen nationalen Identitäten normalerweise, i. e. innerhalb ihrer sozialen Gemeinschaft, als geteiltes Wissen voraussetzen kann und nicht in Worte fassen, hinterfragen oder näher erläutern muss.

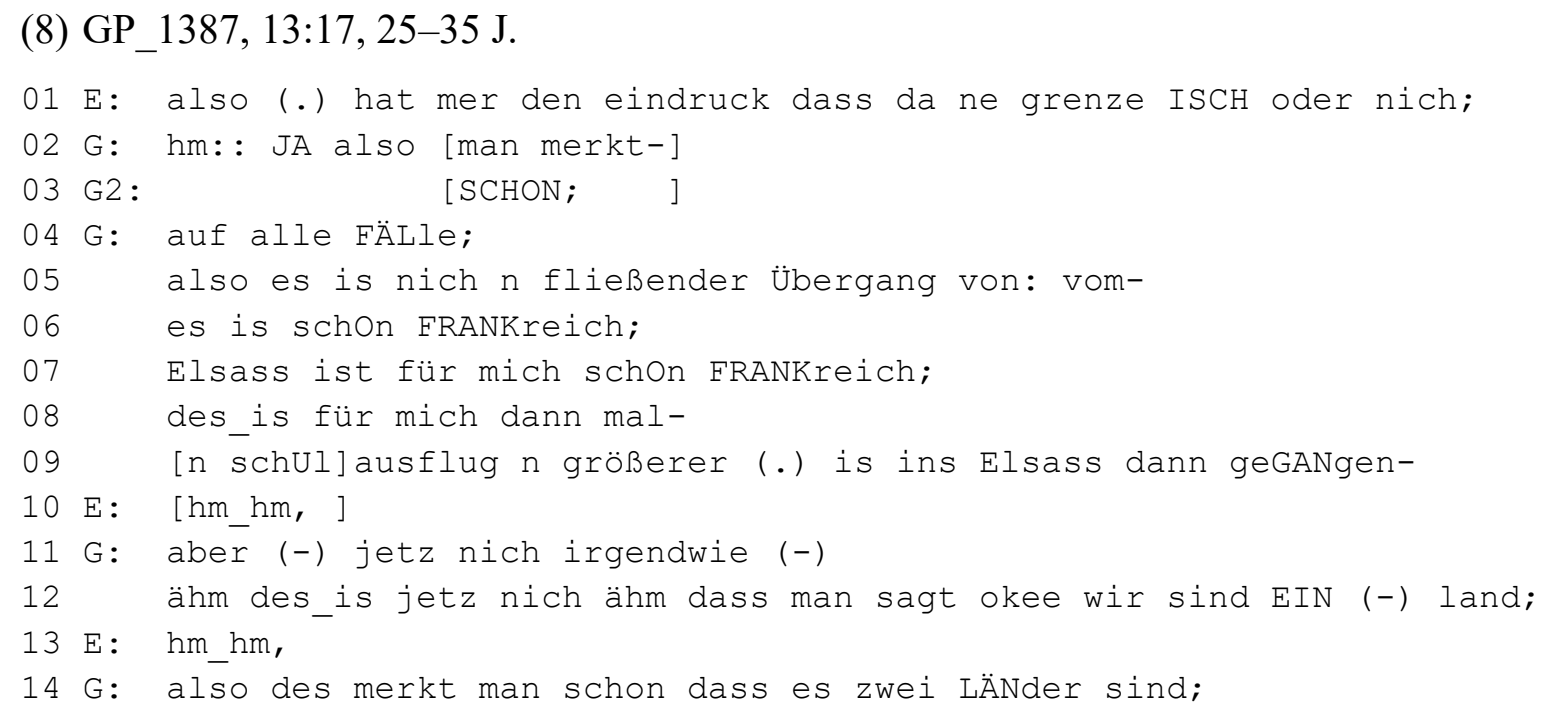




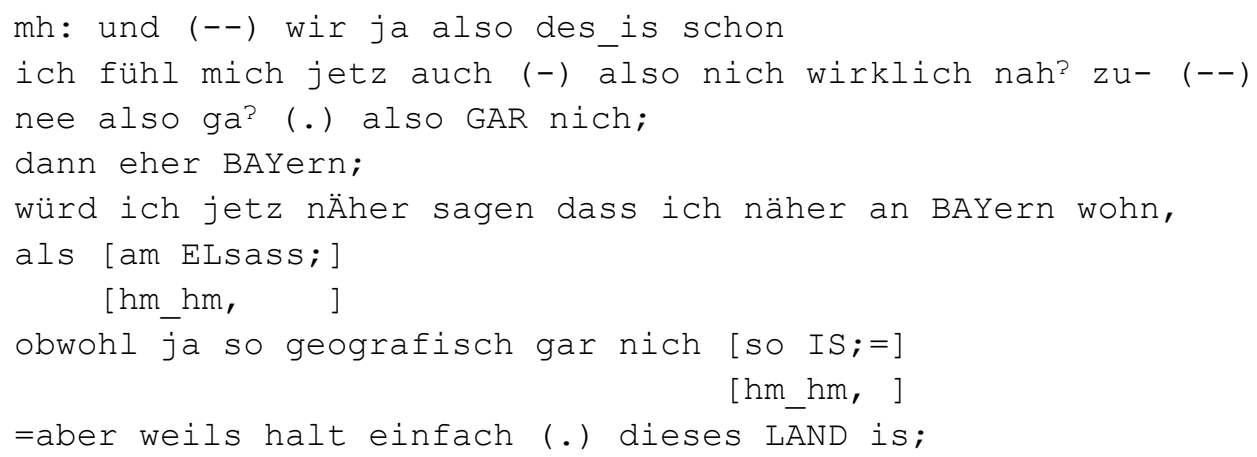

Obwohl die Grenze de facto nicht mehr sichtbar ist, existiert sie für die Informantin dennoch auf alle FÄLle (Z. 04). Es existiere kein fließender Übergang (Z. 05) zwischen Baden und Elsass (bzw. Deutschland und Frankreich), sondern eine „harte“ Grenze, die die Nationalstaaten Deutschland und Frankreich als diskrete Einheiten klar voneinander trennt. Im Zuge der Erläuterung ihrer Sicht auf den Grenzraum konstruiert die Informantin eine nationale Identität. Der Kern dieser Identitätskonstruktion besteht in der Gegenüberstellung der Regionen Elsass und Bayern, die sie in Hinblick auf die von ihr subjektiv empfundene Verbundenheit (sie wählt die Metapher der ,gefühlten Nähe“, Z. 16/19) beurteilt und miteinander vergleicht. Sie kommt zum Ergebnis, dass sie eine größere Nähe zu Bayern empfindet (Z. 19), weil Bayern zu Deutschland gehört (Z. 24). Im Gegensatz dazu fühlt sie sich dem Elsass als Teil Frankreichs nich wirklich nah (Z. 16), also GAR nich (Z. 17), obwohl die geografischen Verhältnisse diesem Urteil eigentlich zuwiderlaufen (Z. 22). Diese Ausführungen belegen, dass die Idee des Nationalstaats für die Identitätskonstruktion dieser Informantin von zentraler Bedeutung ist.

Die nationalstaatliche Ideologie, die in diesen Aussagen zum Vorschein kommt, ist verbunden mit einer Zuordnung von Regionen zu Nationen. Die Regionen Baden und Bayern werden Deutschland zugeordnet und sind auf diese Weise miteinander verbunden (Z. 24); das Elsass wird Frankreich zugeordnet (Z.07). Die Möglichkeit, dass Regionen zu unterschiedlichen Nationalstaaten gehören und dennoch - über die Staatsgrenze hinweg - miteinander verbunden sind, scheint mit dieser Ideologie nicht kompatibel zu sein. Entsprechend tauchen in ihren Überlegungen zum Wesen des Grenzraums auch nur zwei Kategorisierungsalternativen auf: EIN (-) land (Z. 12) - diese Möglichkeit der Zugehörigkeit Badens und des Elsass zu einem Nationalstaat wird aber gleich verworfen - und zwei LÄNder (Z. 14), für die sie sich ausspricht.

Die nationalstaatliche Ideologie, auf die sich diese Informatin stützt, zählt zu den sozialen Repräsentationen, die von den Bewohnern der grenzferneren Städte (und des Markgräflerlands, siehe 3.5) besonders häufig diskursiv aktiviert und zur Identitätskonstruktion herangezogen werden. Solche Positionierungen stehen in Kontrast zur Sichtweise vieler InformantInnen aus den oben behandelten badischen Grenzgebieten (insbesondere Hanauerland und Kaiserstuhl), die oft eine grenzüberschreitende Verbundenheit wahrnehmen und eine durch die Zugehörigkeit zum Oberrheingebiet motivierte, regionale Identität konstruieren. Wie wir oben gesehen haben (siehe (1)), kommen manche dieser Gewährspersonen daher auch zur Einschätzung, dass ihnen die Elsässer näher stehen als Deutsche aus weiter entfernten Gebieten.

Auch andere Gewährspersonen aus den grenzferneren Städten setzen Elsass und Frankreich tendenziell gleich und differenzieren dementsprechend auch nicht zwischen Elsässern und Franzosen. Eine Informantin ist etwa der Meinung, dass Elsässer hinsichtlich ihrer Mentalität 
so seien wie die Franzosen, nämlich „lockerer drauf“ in Bezug auf „Regeln“ oder „Pünktlichkeit“ (Offenburg 1). Es finden sich jedoch auch Gewährspersonen, die sich hinsichtlich des Elsass völlig indifferent zeigen und nichts über den Charakter der Elsässer wissen (z. B. Freiburg 1, Baden-Baden 2).

Diese Befunde deuten darauf hin, dass sich die meisten Gewährspersonen aus den grenzferneren Städten nicht mit dem Elsass verbunden fühlen. Manche sehen jedoch sehr wohl Gemeinsamkeiten zwischen Elsässern und den Bewohnern kleiner badischer Rheindörfer, von denen sie sich aber gleichzeitig sprachlich und charakterlich abgrenzen (Offenburg 5, Achern 7). Dabei zeigt sich insbesondere in Achern, dass sich einige Gewährspersonen eher in Richtung Schwarzwald orientieren als in Richtung Rhein oder gar darüber hinaus, etwa was die Wahrnehmung der eigenen Zugehörigkeit $\mathrm{zu}$ einem bestimmten Lebensraum oder die Gestaltung von Freizeitaktivitäten angeht (Achern 1, Achern 3, Achern 5). Ähnlich wie in Muggensturm (siehe 3.3) könnte hier die relativ große Distanz zum Rhein bzw. die Nähe zum Schwarzwald eine Rolle spielen. Die direkte räumliche Umgebung prägt die Lebenswelt dieser Ortsbewohner und scheint für die Konstruktion ihrer regionalen Identität eine wichtige Rolle zu spielen.

Die InformantInnen (sowohl aus Dörfern als auch aus Städten) sind der Meinung, dass in den Städten der Dialektgebrauch weniger ausgeprägt ist als in den Dörfern, was dem Ergebnis ethnodialektologischer Untersuchungen zu dieser Region entspricht (cf. Stoeckle 2014: 521). Bei der Kommunikation mit den Elsässern weichen die Stadtbewohner in ihrer Sprachwahl sehr stark von den Regionen Hanauerland und Kaiserstuhl, etwas weniger stark auch vom Landkreis Rastatt ab. Die Mehrheit der Gewährspersonen gibt von sich an, im Kontakt mit Elsässern nicht auf das Alemannische zurückzugreifen. Nur eine Minderheit - fast ausschließlich aus der älteren Gruppe - kommuniziert mit Elsässern im Dialekt. In der jüngeren Gruppe verwendet nur eine Person den Dialekt als grenzüberschreitendes Kommunikationsmittel.

Hinsichtlich der Relation des eigenen Dialekts zum Elsässischen sehen die Stadtbewohner sofern sie überhaupt eine Vorstellung vom Elsässischen haben - keine große Ähnlichkeit. Stattdessen schreiben sie dem benachbarten Hanauerland (Offenburg 7, Achern 5, Achern 7) und dem benachbarten, südlich des Hanauerlandes gelegenen Ried (Offenburg 3, Offenburg 4, Offenburg 5) einen Dialekt zu, der stärker ist als ihr eigener und dem Elsässischen nahesteht. Die Stadtbewohner grenzen sich oft stark von der Sprechweise der Dörfer im Ried und Hanauerland $\mathrm{ab}$ und schreiben diesen Rheindörfern gleichzeitig eine größere sprachliche (und charakterliche) Nähe zu den Elsässern zu, wodurch sie sich auch vom Elsass abgrenzen (z. B. „Elsässisch ist komplett anders, schon Freistett [Hanauerland] schwätzt einen Dialekt, den ich nicht verstanden hab“, Achern 3; „Das Ried ist für mich fast nicht mehr Ortenau sondern eine Pufferzone [zum Rhein/Elsass]“, Offenburg 4). ${ }^{20}$ Die Gewährspersonen aus den Städten bekunden insgesamt auch, deutlich größere Verständnisprobleme mit dem Elsässischen zu haben als die Gewährspersonen aus den rheinnahen Dörfern, die in den vorangehenden Abschnitten behandelt wurden. Teilweise konstruieren die Gewährspersonen diesen Kontrast in ihren Aussagen selbst, wie im folgenden Ausschnitt. Hier erläutert die Informantin die

\footnotetext{
${ }^{20}$ Eckige Klammern in Zitaten markieren inhaltliche Ergänzungen des Autors, die zum besseren Verständnis vorgenommen wurden.
} 
sprachliche Nähe des an der nördlichen Grenze des Hanauerlands in Rheinnähe gelegenen Dorfs Lichtenau-Ulm zum Elsass:

(9) GP_1303, 49:02, 60-70 J.

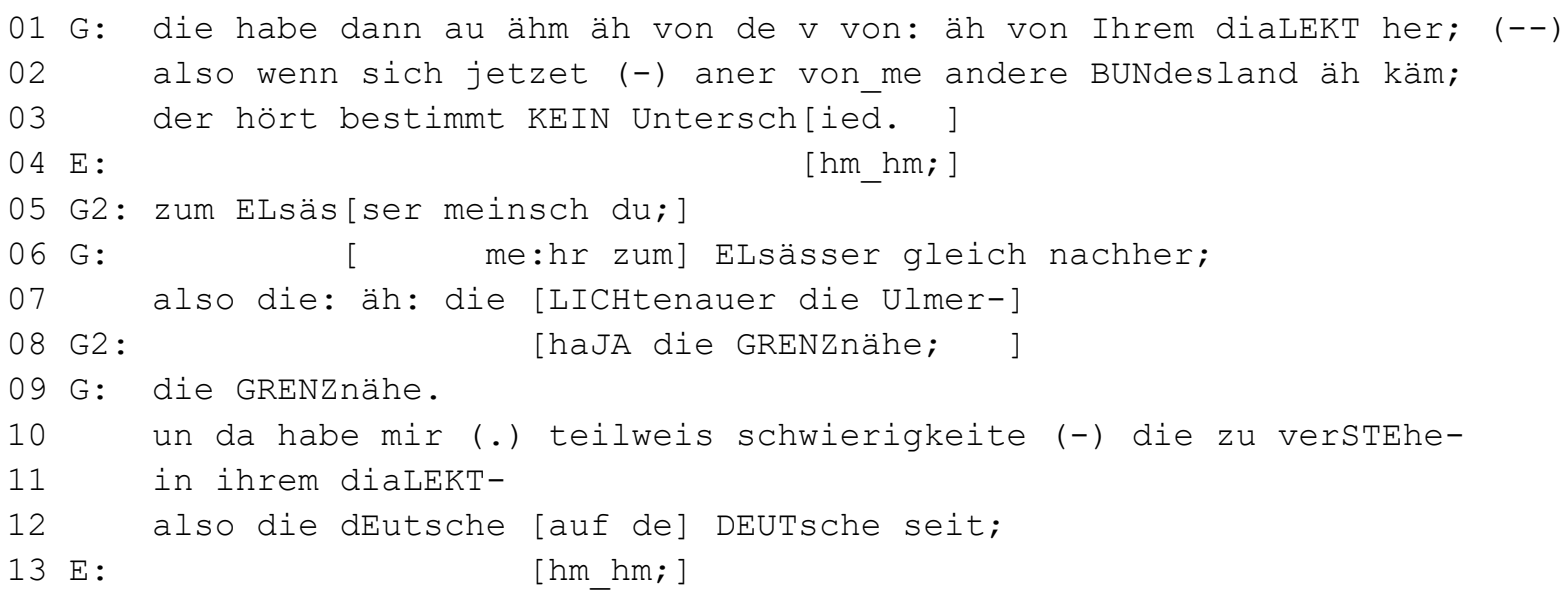

Diese Informantin empfindet einen großen Unterschied zwischen ihrem eigenen Dialekt und dem Elsässischen. Zur Verdeutlichung, wie eklatant dieser Unterschied ist, führt sie das Beispiel eines Dialekts aus dem Hanauerland an. Der Dialekt des auf der badischen Rheinseite gelegenen Dorfs Lichtenau-Ulm sei dem Elsässischen so ähnlich, dass Außenstehende keinen Unterschied zum Elsässischen wahrnehmen könnten (Z.02/03). Die Konstruktion dieser Parallele setzt die rheinnahen badischen Dialekte in diesem Gebiet tendenziell mit dem Elsässischen gleich. Die Informantin fährt fort, sie habe teilweis schwierigkeite (Z. 10), den Dialekt auf de DEUTsche seit (Z. 12) zu verstehen und betont im weiteren Gesprächsverlauf, dass dieser Dialekt der „stärkste Dialekt“ in der Umgebung und ihr „fremd“ sei. Indem sie dem Explorator vor Augen führt, dass zwischen ihrem eigenen Dialekt und dem Elsässischen noch andere badische Dialekte, die dem Elsässischen viel ähnlicher sind, gewissermaßen als Zwischenstufen liegen, konstruiert die Gewährsperson eine besonders große Distanz zwischen ihrem Dialekt und dem Elsässischen als einem teilweise unverständlichen Dialekt.

Neben der Zuschreibung dialektaler Differenz, die sich in vergleichbarer Weise bei vielen anderen InformantInnen aus grenzferneren Städten findet, zeigen die Aussagen auch, dass diese Personen weniger in Kontakt mit dem Elsässischen kommen als die Bewohner der oben behandelten Regionen. Manche Gewährspersonen geben von sich an, das Elsässische nicht zu kennen (Offenburg 6). Andere sagen aus, sich nicht an das Elsässische zu erinnern oder halten gar Elsässisch und Französisch für dasselbe (Achern 1).

Insgesamt zeigt sich ein deutlicher Kontrast zwischen den Gewährspersonen aus den grenzferneren Städten einerseits und aus den drei oben behandelten Regionen andererseits, insbesondere dem Hanauerland und dem Kaiserstuhl. Die schwächere Verbundenheit zeigt sich auf allen drei untersuchten Ebenen: Die Stadtbewohner sehen weniger häufig grenzüberschreitende regionale, kulturelle oder charakterliche Gemeinsamkeiten, wählen seltener den Dialekt für die grenzübergreifende Kommunikation und haben größere Verständnisprobleme mit dem Elsässischen. 


\subsection{Markgräflerland}

Der folgende Abschnitt analysiert die Identitätskonstruktionen der (ausschließlich jüngeren, siehe Beginn Abschnitt 2) Gewährspersonen aus den Erhebungsorten Weil, Holzen, Neuenburg und Staufen, die in der heute als Markgräflerland bezeichneten Region am südlichen Oberrhein liegen. Diese Region verdankt ihren Namen der historischen Zugehörigkeit zur „Oberen Markgrafschaft" (Hoffmann 1994: 224), die sich etwa zu Beginn des 12. Jahrhunderts durch die Herrschaft Rötteln allmählich herauszubilden begann, sich durch die Zusammenkunft der Herrschaften Rötteln und Hachberg-Sausenberg im Jahre 1315 erweiterte und durch die Vereinigung mit der Herrschaft Badenweiler im Jahre 1444 ihre größte Ausdehnung erreichte (cf. Wörner 1994).

Es spricht vieles dafür, dass die Bewohner des Markgräflerlandes in Richtung Süden orientiert waren, wo nicht weit entfernt die Stadt Basel liegt, die in der Region eine enorme politische, kulturelle und wirtschaftliche Bedeutung hatte und aus historischer Perspektive sogar als „natürlicher Mittelpunkt“ (Wörner 1994: 65) oder „,so etwas wie die Hauptstadt“ (Hoffmann 1994: 241) des Markgräflerlands betrachtet werden kann. Auch in sprachlicher Hinsicht zeigen sich in der Zugehörigkeit zum Südalemannischen (und nicht wie die nördlicher gelegenen Gebiete zum Oberrheinalemannischen) die südlichen Einflüsse auf das Markgräflerland. Die Beziehungen ins Elsass scheinen in dieser Region hingegen weniger stark ausgeprägt gewesen zu sein als die Beziehungen zur Schweiz und insbesondere zu Basel. Dafür spricht auch Maurers Beobachtung (1942: 281, 284), dass sich gerade dort, wo die politische Grenze der Oberen Markgrafschaft entlang des Rheins verlief, eine der wenigen Stellen findet, an denen der Rhein „auf kurze Strecken Sprachgrenze“ (ibd.: 281) sei.

Auch in den Interviews zeichnet sich dieses Bild ab. Die Gewährspersonen können zwar insgesamt kaum Aussagen über das Elsass machen, erwähnen aber in ihren Ausführungen zu grenzübergreifenden Alltagsaktivitäten und zur Sprache im Grenzraum mehrfach Basel und die Schweiz als Bezugspunkte: So sagt etwa ein Informant aus, dass man „Basel Stadt-Land“ noch mit zum Markgräflerland zählen könne (Weil 2), was die Sichtweise der oben zitierten historischen Arbeiten stützt. Verschiedene InformantInnen aus den beiden südlichsten Erhebungsorten fahren ,lieber in die Schweiz als nach Frankreich“ (Holzen 1, Weil 1), besuchen in Basel kulturelle Veranstaltungen (Weil 2), „wüssten mehr“ über die Schweiz als über das Elsass (Weil 1) oder heben positiv hervor, dass die Schweizer - anders als die Deutschen - so stolz auf ihren Dialekt seien. Zudem schätzen diese Gewährspersonen ihren eigenen Dialekt tendenziell als dem Schweizerdeutschen ähnlich ein. Ein Informant gibt sogar von sich an, schon einmal aufgrund seines Dialekts für einen Schweizer gehalten worden zu sein (Holzen 2). Diese Beobachtungen deuten darauf hin, dass die Gewährspersonen aus dem Markgräflerland zwar keine engen Beziehungen ins Elsass sehen, aber stattdessen - vor allem in den südlichen Orten - eine gewisse regionale und sprachliche Verbundenheit mit der Schweiz.

Wie stark die schweizerisch-deutsche Grenze im Vergleich zur französisch-deutschen Grenze im Bewusstsein mancher Gewährspersonen präsent ist, zeigt sich im folgenden Ausschnitt. Hier war die Grenze zu Frankreich bereits zuvor Gesprächsgegenstand. Der Informant hatte deutlich 
gemacht, dass er keinen Bezug zu Frankreich habe und nicht dorthin fahre. Zu Beginn des Ausschnitts greift die Exploratorin dieses Thema mit „,rüberfahren“ wieder auf:

$$
\text { GP_1441, 15:19, 25-35 J. }
$$

$01 \mathrm{E}$ : un: also du fahrsch dann GAR net rüber;

$02 \mathrm{G}:{ }^{\circ} \mathrm{h}(-)$ ja: (-) bAsel hat einiges an muSEen,

03 DA halt ab [und zu- ]

$04 \mathrm{E}: \quad[$ ah BAsel; $]$

05 aber jetz nit (.) noch FRANKriich;

06 G: nein frAnkreich NICHT.

$07 \mathrm{E}:$ ah: OKEE;

08 also scho de: (-) du orientIersch dich dann eher richtung SCHWIZ;

$09 \mathrm{G}: j \mathrm{ja}$

Obwohl das „Rüberfahren“ im Gesprächsverlauf zuletzt mit Bezug zur französisch-deutschen Grenze behandelt wurde, löst die Frage in Z. 01 beim Informanten unmittelbar die Assoziation des Grenzübertritts in die Schweiz aus (siehe die Erwähnung von Basel in Z. 02). Die Reaktion der Exploratorin zeigt, dass diese Antwort neue Information liefert und von der erwarteten Antwort abweicht (siehe den Erkenntnisprozessmarker $a h$ in Z.04 und die verständnissichernde Kontrastierung aber jetz nit (.) noch FRANKriich in Z. 05). In der folgenden Sequenz sichert die Exploratorin die Erkenntnis ab, dass der Informant sich eher in Richtung Schweiz als nach Frankreich orientiert.

Der fehlende Bezug dieses Informanten zu Frankreich bzw. zum Elsass kommt auch darin zum Ausdruck, dass er kein richtiges Bild von „den Elsässern“ hat. Auf die Frage nach dem Charakter der Elsässer kann er nichts sagen, weil er keine kennt. Daraufhin fragt ihn die Exploratorin, ob er eine Vorstellung vom ,prototypischen Elsässer“" habe:

(11) GP_1441, 15:19, 25-35 J.

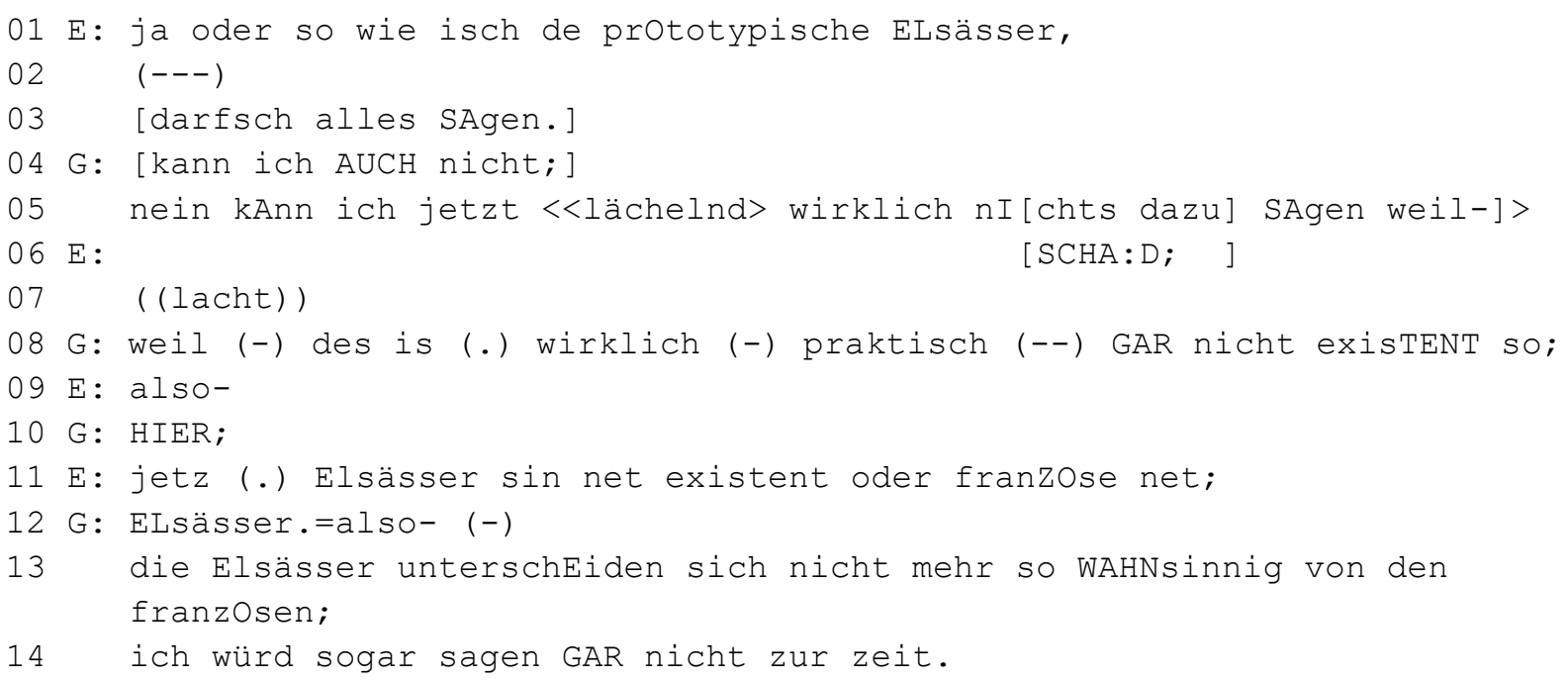

Da der Informant bereits angegeben hat, nicht über persönliche Erfahrungen mit Elsässern zu verfügen, kann die Frage, wie der prOtotypische ELsässer (Z. 01) sei, nur auf tradiertes, sozial geteiltes Wissen abzielen, etwa in der Form von Stereotypen. Es liegen verschiedene Anhaltspunkte dafür vor, dass die Interagierenden die Frage auch in diesem Sinne verstehen, nämlich als Eröffnung der Möglichkeit, heikle Meinungen über die Elsässer kundzutun, die möglicherweise nicht der political correctness entsprechen (darfsch alles SAgen in Z.03, 
Lächeln in Z. 05, SCHA:D in Z. 06, Lachen in Z. 07). Auch eine solche Antwort scheint dem Informanten jedoch schwer zu fallen, wie die lange Pause vor (Z. 02) und die vielen Pausen innerhalb seiner Äußerung (Z. 08) zeigen. Der Grund dafür, dass er auf diese Frage nicht antwortet, scheint nicht etwa darin zu bestehen, dass ihm die Nennung von negativen Stereotypen unangenehm ist. (Diese Vermutung wird dadurch unterstützt, dass er dies an anderer Stelle im Gespräch bezüglich „der Franzosen“ tut.) Vielmehr weist er darauf hin, dass er überhaupt nicht in der Lage ist, eine Antwort zu geben, weil die Elsässer HIER (Z. 10) GAR nicht exisTENT seien (Z. 08). Mit dem Lokaladverb „hier“ verweist er dabei auf seinen Wohnort bzw. auf eine nicht näher spezifizierte Gegend, in der er lebt. Die Herstellung dieser Referenz impliziert, dass die „Nicht-Existenz“ der Elsässer aus seiner Sicht lokal begrenzt sein könnte, dass also die Elsässer möglicherweise in anderen Gegenden als Teil der Lebenswelt existieren. Am Ende des Ausschnitts verdeutlicht der Informant, wie seine Auffassung von der „Nicht-Existenz“ der Elsässer zu verstehen ist - nämlich in dem Sinne, dass sie sich GAR nicht (Z. 14) von den franZOsen (Z. 13) unterscheiden.

Die Positionierung dieses Informanten steht exemplarisch für die (allesamt jüngeren) InformantInnen aus dem Markgräflerland. Aus dieser Personengruppe - anders als die jüngeren Gewährspersonen aus dem Hanauerland und vom Kaiserstuhl und teilweise aus dem Landkreis Rastatt - konstruiert niemand eine grenzüberschreitende Zusammengehörigkeit. Stattdessen bringen diese InformantInnen eine indifferente Haltung der anderen Seite gegenüber zum Ausdruck, ohne auf persönliche Erfahrungen im Umgang mit Elsässern zurückgreifen zu können. Oft wird nicht, wie in (11), zwischen Franzosen und Elsässern unterschieden. Die nationalen Kategorien (Deutsche vs. Franzosen) und national motivierte Abgrenzungen, häufig in Form stereotypischer Zuschreibungen, stehen im Vordergrund.

Der mangelnde Bezug zum Elsass, der unter den InformantInnen aus dem Markgräflerland tendenziell vorherrscht, zeigt sich auch im Kontext von Interviewfragen über die sprachlichen Verhältnisse im Grenzraum. Nur eine Person gibt an, das Elsässische zu verstehen und sieht im Elsässischen einen alemannischen Dialekt mit französischem Akzent (Neuenburg 1). Eine andere Informantin kennt das Elsässische zwar nicht, stellt aber Vermutungen darüber an, dass sich der eigene Dialekt vom Elsässischen aufgrund der unterschiedlichen „Ursprünge“ des Deutschen und Französischen aller Wahrscheinlichkeit nach unterscheiden müsste (Weil 1). Alle übrigen haben überhaupt keine Vorstellung vom Elsässischen. ${ }^{21}$ Sie können daher weder Aussagen zum Verständnis des Elsässischen treffen noch, wie der nächste Ausschnitt zeigt, ihren eigenen Dialekt in Relation zum Elsässischen setzen:

$$
\text { GP_1441, 13:14, 25-35 J. }
$$

$01 \mathrm{E}$ : oder froge mer amol WELche site;

02 also ischs de gliiche dialekt wie in der SCHWIZ,

03 oder ischs de gliiche dialekt wie in: FRANKriich?

\footnotetext{
${ }^{21}$ Dies gilt nicht für einen älteren Informanten aus dem Markgräflerland, der dem Elsässischen eine Nähe zum Kaiserstühler Dialekt zuschreibt, nicht aber zum eigenen. Obwohl die älteren Gewährspersonen aus dem Markgräflerland in diesem Beitrag ansonsten nicht berücksichtigt werden (siehe Beginn Abschnitt 3), wird hier auf diese Beobachtung hingewiesen, da sie einen zusätzlichen Beleg für die in dieser Region häufig anzutreffende Konstruktion von Alterität in Bezug auf die andere Rheinseite liefert.
} 


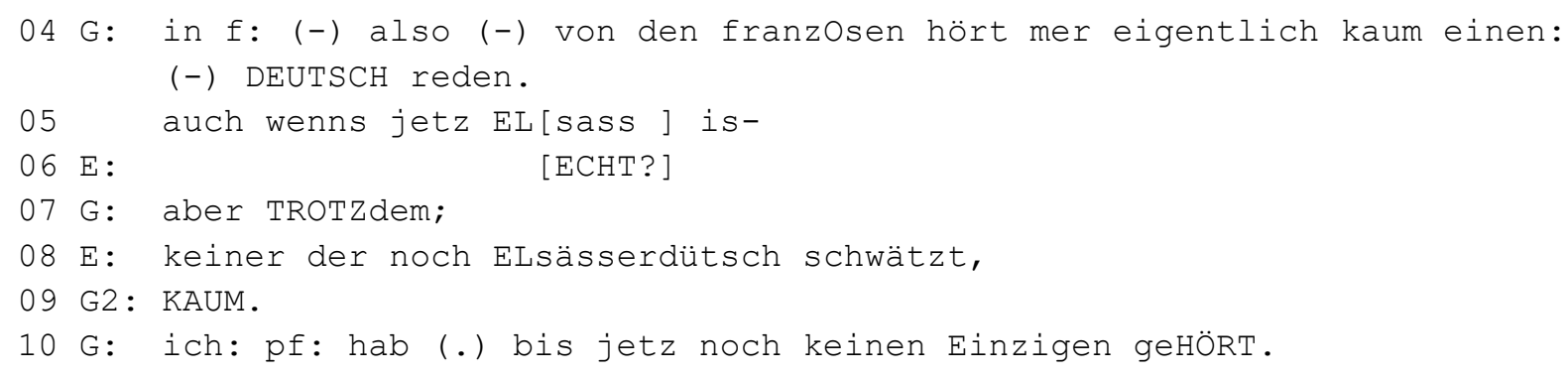

Auf die Frage der Exploratorin, ob es sich auf der deutschen und der französischen (und Schweizer) Seite des Rheins um denselben Dialekt handle (Z. 01-03), gibt der Informant keine darauf bezogene Einschätzung als Antwort ab. Vielmehr weist er darauf hin, dass er Elsässisch noch nie gehört habe (Z. 10), wodurch sich eine Antwort auf die eigentliche Frage erübrigt. Auch die Mutter des Informanten (G2) ist der Meinung, dass KAUM (Z. 09) noch jemand Elsässisch spreche.

Da unter den Gewährspersonen kaum jemand eine Vorstellung vom Elsässischen hat, ist es auch nicht weiter verwunderlich, dass in der grenzüberschreitenden Kommunikation fast niemand auf den Dialekt zurückgreift. Nur eine Person gibt an, Dialekt im Elsass zu sprechen (und zwar nur in dem Restaurant, in dem sie regelmäßig isst, Neuenburg 1), die übrigen setzen entweder andere kommunikative Mittel ein („Hochdeutsch“, Französisch, Englisch oder „Hände und Füße“) oder geben an, gar nicht mit Elsässern zu sprechen:

$$
\text { GP_1481, 11:28, 25-35 J. }
$$

$01 \mathrm{E}$ : ich WEISS ja net; =

02 =hend ihr scho mol im dialekt mit_eme Elsässer gSCHẄ̈TZT?=oder-

$03 \mathrm{G}$ : [ich hab] mich no NIE unterhalte mit [eim; ]

$04 \mathrm{G} 2$ : [hm_hm; ] [hm_hm; ]

$05 \mathrm{G}: \mathrm{NEE}$,

$06 \mathrm{E}:$ äh (-) wIsst ihr ungefähr wie so der elsässische dialekt ISCH?

07 [dann frog ich] [mol so, ]

08 G2: [((lacht)) ] [net WIRK]lich; [( lacht)) ]

$09 \mathrm{G}: \quad$ [Au net WIRKlich;=nai, ]

Die Informantin (G) und der Informant (G2), die gemeinsam an diesem Interview teilnehmen, verfügen weder über kommunikative Erfahrungen mit Elsässern (Z. 03-05) noch haben sie eine ungefähre Vorstellung vom Elsässischen (Z. 08/09). Auch dieser Ausschnitt fügt sich also in das Gesamtbild ein, das sich in den anderen Beispielen für das Markgräflerland abzeichnet: Kontakte mit den elsässischen Nachbarn scheinen sehr selten zu sein.

Zusammenfassend lässt sich sagen, dass die jüngeren InformantInnen aus dem Markgräflerland keine grenzüberschreitende Verbundenheit mit dem Elsass sehen, größtenteils auf keine kommunikativen Erfahrungen mit Elsässern zurückgreifen können und daher zumeist keine Vorstellung vom Elsässischen haben. Im Gegensatz zu vielen jüngeren Gewährspersonen aus den anderen Regionen endet die Lebenswelt fast aller jüngeren Gewährspersonen aus dem Markgräflerland an der französisch-deutschen Grenze.

\section{$4 \quad$ Abschließende Diskussion}

Ziel des Beitrags war eine Bestandsaufnahme der grenzüberschreitenden Identitäten im badischen Oberrheingebiet. Dafür wurden die interaktionalen Positionierungen von 127 
InformantInnen zu drei Themenbereichen untersucht: 1) regionale Verbundenheit mit dem Elsass, 2) Sprachwahl in der grenzüberschreitenden Kommunikation und 3) Verständnis des Elsässischen. Die qualitative Analyse hat gezeigt, dass sich die Identitätskonstruktionen je nach Teilregion deutlich unterscheiden. Es ergibt sich folgendes Gesamtbild hinsichtlich der Verbundenheit der einzelnen Regionen mit dem Elsass (absteigend von stark bis schwach):

Hanauerland $>$ Kaiserstuhl $>$ Landkreis Rastatt $>$ grenzfernere Städte $>$ Markgräflerland.

Das Hauptergebnis, das in dieser Rangfolge zum Ausdruck kommt, besteht darin, dass die historischen Verhältnisse einer Region, vor allem deren politische Geschichte, die Identitätskonstruktionen der dort ansässigen Personen auch heute noch entscheidend prägen. Die Region Hanauerland, die über mehrere Jahrhunderte Teil eines Herrschaftsgebiets war, zu dem vor allem linksrheinisches Gebiet zählte, hatte von allen im vorliegenden Beitrag untersuchten Regionen die engsten historischen Beziehungen zum Elsass. Die Gewährspersonen aus dem Hanauerland sehen auch heute noch am ehesten eine gemeinsame Identität mit den Bewohnern der anderen Rheinseite. Für den Kaiserstuhl und den Landkreis Rastatt lassen sich in der Geschichte zwar ebenfalls Verbindungen zum Elsass ausmachen, allerdings sind die historischen politischen Beziehungen dieser Regionen zum Elsass als weniger eng einzuschätzen. Dem entsprechen die grenzüberschreitenden Identitäten, die die InformantInnen vom Kaiserstuhl und aus dem Landkreis Rastatt konstruieren. In beiden Regionen wird eine etwas weniger starke Verbundenheit zum Elsass wahrgenommen als im Hanauerland, wobei der Kaiserstuhl sich vor allem hinsichtlich der Konstruktion größerer sprachlicher Nähe vom Landkreis Rastatt abhebt.

Gerade diejenigen Gewährspersonen, die in den drei erwähnten Regionen eine enge Beziehung zum Elsass sehen, verfügen häufig noch über Wissen um die historischen Verbindungen zum Elsass und bringen dieses immer wieder ins Gespräch ein (z. B. historische Zugehörigkeit des Wohnorts zum Elsass, früherer linksrheinischer Landbesitz ortsansässiger Familien, Verkauf landwirtschaftlicher Produkte auf dem Markt in Straßburg durch Vorfahren, etc.). Noch deutlicher kommt die Nähe zum Elsass jedoch in den Identitätskonstruktionen der Gewährspersonen zum Ausdruck, die nicht explizit auf die historischen Verhältnisse Bezug nehmen, diese jedoch implizit widerzuspiegeln scheinen. Insbesondere im Hanauerland, teilweise aber auch am Kaiserstuhl und im Landkreis Rastatt, weisen viele Gewährspersonen auf die ähnliche Wesensart der Elsässer hin: Sie seien von der Mentalität her „,mehr Deutscher als Franzose“, also „,schaffig“, ,geradlinig“, „pünktlich“ und „ehrlich“- besäßen also ,,deutsche Tugenden“" (siehe (3)). Insgesamt zeigen diese Aussagen deutlich, dass wichtige Punkte der Autostereotypisierung auf die Elsässer übertragen werden. Neben diesen Eigenschaften, die die Elsässer mit den Deutschen teilen, werden aber auch Unterschiede genannt: Elsässer „leben gerne“, sind „Genießer“, „kulinarisch fortschrittlich“, „gemütlicher“, „offener“, „lockerer“, „unbeschwerter“. Viele dieser Charakterzüge werden in den Interviews auch den Franzosen zugeschrieben, so dass man in diesen Fällen von einer positiven Darstellung „des Elsässers“ sprechen kann, die positive stereotypische Merkmale „des Deutschen“ und „des Franzosen“ verbindet. Diese Sicht auf die Elsässer ähnelt dem Bild der Elsässer von sich selbst (cf. Auer 2018). Es gibt also entweder in diesen Regionen des Oberrheinsgebiets heute noch Bereiche der Lebenswelt, die die Bewohner beider Rheinseiten miteinander teilen, oder dies war 
zumindest früher einmal der Fall und die Einheimischen geben ihr tradiertes Wissen als soziale Repräsentationen von Generation zu Generation weiter.

Die Identitätskonstruktionen der Gewährspersonen aus den grenzferneren Städten und dem Markgräflerland unterscheiden sich insgesamt deutlich von den drei anderen Regionen, wobei jeweils unterschiedliche Gründe für die Wahrnehmung einer schwächeren grenzüberschreitenden Verbundenheit oder gar die völlige Bezugslosigkeit zum Elsass plausibel erscheinen. Im Falle der Städte sind wohl vor allem ihr Status als eigenständige Zentren, ihre Zugehörigkeit zu historischen Territorien mit eher schwacher Orientierung in Richtung Elsass und auch ihre größere Distanz zum Rhein (siehe unten) ausschlaggebend; im Falle des Markgräflerlands scheint der Hauptgrund in der historischen Orientierung in Richtung Basel zu liegen.

Über alle Regionen hinweg gilt, dass die Aussagen der einzelnen Gewährspersonen zu den drei Themenbereichen regionale Verbundenheit mit dem Elsass, grenzüberschreitende Sprachwahl und Verständnis des Elsässischen tendenziell in dieselbe Richtung weisen. Wichtig hervorzuheben ist insbesondere der Zusammenhang zwischen der regionalen Verbundenheit und der Sprachwahl: Personen, die eine enge regionale Verbundenheit mit dem Elsass sehen, setzen tendenziell (zumindest in manchen Situationen) den Dialekt in der grenzübergreifenden Kommunikation ein, wohingegen Personen, die keine solche Verbundenheit sehen, den Dialekt in der Regel nicht einsetzen. In den einzelnen Regionen bestehen dementsprechend folgende Zusammenhänge: Während bei einer typischen Gewährsperson aus dem Hanauerland eine starke regionale Verbundenheit mit dem Elsass zumeist mit der Wahl des Dialekts in der grenzüberschreitenden Kommunikation und einem problemlosen Verständnis des Elsässischen in Verbindung steht, fällt beim typischen Stadtbewohner die schwache Verbundenheit mit dem Elsass häufig mit der Wahl anderer grenzübergreifender Kommunikationsressourcen als dem Dialekt und Verständnisproblemen mit dem Elsässischen zusammen. Im Markgräflerland führt der fehlende Bezug zum Elsass bzw. die fehlende kommunikative Erfahrung mit Elsässern sogar größtenteils zur Unmöglichkeit, eine Verbundenheit mit „den Anderen“ überhaupt in Betracht zu ziehen oder Aussagen über deren Sprache zu treffen. Der Kaiserstuhl und der Landkreis Rastatt liegen zwischen diesen Extrempolen, wobei hier die Identitätskonstruktionen der Mehrheit der Gewährspersonen eher dem Hanauerland ähneln, die einer Minderheit eher denen der Stadtbewohner.

Neben der historischen Verbundenheit mit dem Elsass übt auch die g e grafis che Nähe zum Rhein einen bedeutenden Einfluss auf die Identitätskonstruktionen aus. Je näher ein Ort an der französisch-deutschen Grenze liegt, desto enger wird seine Verbundenheit mit dem Elsass konstruiert, und zwar sowohl in der Selbstzuschreibung (Aussagen der Bewohner rheinnaher Orte über sich selbst) als auch in der Fremdzuschreibung (Aussagen von Gewährspersonen aus rheinferneren Orten über Bewohner rheinnaher Orte). Zuweilen wird auf diese Weise eine Verbundenheit auf der Ebene der Wesensart hergestellt, in den meisten Fällen betrifft die Konstruktion von Verbundenheit durch räumliche Nähe zum Rhein aber die Ebene der Sprache (siehe (9)). Unter den badischen Gewährspersonen ist die Einschätzung insgesamt weit verbreitet, dass der Unterschied zwischen den rechtsrheinischen alemannischen Dialekten und dem Elsässischen mit zunehmender Distanz zum Rhein größer wird. Die subjektiv wahrgenommenen Verständnisprobleme mit dem Elsässischen werden zudem tendenziell 
stärker und die Wahl des Dialekts für die grenzüberschreitende Kommunikation seltener, je weiter ein Erhebungsort vom Rhein entfernt ist. Diese Befunde weisen in dieselbe Richtung wie die dialektologischen Ergebnisse, zu denen Schrambke (1981) auf der Grundlage der Daten des Südwestdeutschen Sprachatlas (Steger et al. 1989-2011) kommt. Ihre Analysen zeigen, dass die Ähnlichkeit der links- und rechtsrheinischen alemannischen Dialekte mit zunehmender Distanz zum Rhein immer weiter abnimmt (cf. Schrambke 1981: 7). Die vorliegenden Ergebnisse entsprechen also sowohl der linguistischen Kontinuums-Konzeption als auch dem Alltagsmodell ,je näher, desto ähnlicher, je ferner, desto verschiedener'.

Die Analysen der sprachbezogenen Identitätskonstruktionen ermöglichen auch allgemeinere Einblicke in die ethnodialektologische Gliederung der rechtsrheinischen alemannischen Dialekte in Bezug auf deren Nähe zum Elsässischen. Hier spielen neben der geografischen Nähe zum Rhein die oben behandelten badischen Regionen und der Kontrast Stadt vs. Land eine Rolle. So wie dem Hanauerland vom angrenzenden Landkreis Rastatt und den grenzferneren Städten Achern und Offenburg dialektale Ähnlichkeit mit dem Elsass zugeschrieben wird, so wird dem Kaiserstuhl vom benachbarten Markgräflerland, dem Ried (das in Abschnitt 3.1 gemeinsam mit dem Hanauerland behandelt wurde) von der nahegelegenen Stadt Offenburg und ganz allgemein grenznäheren Orten von grenzferneren Orten sprachliche Nähe zum Elsass zugeschrieben. Die Dialekte im Hanauerland, im Ried und am Kaiserstuhl sind aus Sicht der Gewährspersonen dem Elsässischen am ähnlichsten. Wie oben ausgeführt, sind das genau diejenigen Regionen, die selbst die größte Verbundenheit mit dem Elsass konstruieren und auch objektiv am stärksten mit dem Elsass verbunden waren (das Hanauerland in historischpolitischer, der Kaiserstuhl in dialektologischer Hinsicht). Stoeckles (2014: 519) Ergebnis, dass topografische und territoriale Grenzen eine wichtige Rolle bei der Konstruktion ethnodialektaler Räume spielen, bestätigt sich hier. Das Hanauerland, das außerhalb des von Stoeckle untersuchten Gebiets liegt, scheint insofern einen Sonderfall darzustellen, als es in seiner Untersuchung nur einen vergleichbaren eindeutigen Beleg (den „Hotzenwald“) dafür gibt, dass ein historisches Territorium bei der Konstruktion eines subjektiven Dialektgebiets als salientestes Merkmal herangezogen wird (cf. Stoeckle 2014: 518).

Die Dimensionen „historisch mit dem Elsass verbunden“, „grenznah“ und „dörflich“ scheinen also für die Zuschreibung von sprachlicher Nähe zum Elsass ausschlaggebend zu sein. Da die Dialekte der grenznahen Rheindörfer tendenziell auch als besonders stark eingestuft werden, kann man aus einer etwas anderen Perspektive zusammenfassen: Diejenigen Dialekte, denen der höchste Dialektalitätsgrad zugeschrieben wird, werden als dem Elsässischen besonders ähnlich wahrgenommen.

Die InformantInnen stützen sich in ihren Identitätskonstruktionen stark auf die ihnen zur Verfügung stehenden sozialen Repräsentationen, also die Vorstellungen, die von den Mitgliedern ihrer sozialen Gemeinschaft geteilt werden. Die sozialen Repräsentationen werden in den Interviews dadurch „sichtbar“, dass bestimmte Aussagen innerhalb einer Gruppe von Gewährspersonen (z. B. den Bewohnern einer Region) über viele Interviews hinweg in ähnlicher Form anzutreffen sind, wie etwa die Vorstellung vom „Elsässer mit deutschen Tugenden“, siehe (3). Die Tatsache, dass dieser Topos so häufig diskursiv aktiviert wird, obwohl viele Gewährspersonen gleichzeitig angeben, kaum über persönliche Erfahrungen im Umgang mit Elsässern zu verfügen, spricht für seinen Status als soziale Repräsentation. 
Zusätzlich können in manchen Fällen auch Probleme bei der Versprachlichung darauf hindeuten, dass die InformantInnen einen Ausschnitt aus dem gesellschaftlich verfügbaren Wissensvorrat ansteuern. Dies kann dann der Fall sein, wenn es sich um eine soziale Repräsentation handelt, die anders als die oben erwähnten stereotypen Zuschreibungen in der Regel nicht explizit versprachlicht, sondern von den Mitgliedern einer sozialen Gemeinschaft implizit als selbstverständlich vorausgesetzt wird, wie etwa das Wissen um die Existenz einer Staatsgrenze zwischen Deutschland und Frankreich und die dadurch bedingte nationale Identität, siehe (8). Letztere Fälle treten nicht zuletzt auch deswegen auf, weil die ExploratorInnen als Teil ihrer Rolle innerhalb des soziolinguistischen Interviews immer wieder gezielt das Selbstverständliche hinterfragen, sich als unwissend positionieren oder dort nachhaken, wo aus Sicht der Gewährspersonen das ,natürliche“ Ende einer Gesprächssequenz erreicht wäre.

Ganz allgemein deuten die Ergebnisse darauf hin, dass die sozialen Repräsentationen, die in der badischen Grenzregion am Oberrhein von den ortsansässigen Dialektsprechern als Bestandteil grenzüberschreitender Identitäten geteilt werden, sehr stabile Gebilde sind, die über viele Generationen hinweg erhalten bleiben können. Dennoch zeigt sich auch in den - historisch wie heute - am engsten mit dem Elsass verbundenen Regionen, dass die mit dem Elsass geteilte Identität langsam verblasst und einer nationalen Identität weicht. Dies lässt sich daran ablesen, dass auch das Alter der Gewährspersonen, das in diesem Beitrag nicht im Fokus stand, für die Konstruktion von Verbundenheit mit dem Elsass eine Rolle spielt. Über alle Regionen hinweg sehen ältere Gewährspersonen häufiger eine gemeinsame Identität mit dem Elsass als jüngere (cf. die quantitative Auswertung und die Berechnung eines „Verbundenheitsindex“ für jede Gewährsperson in Pfeiffer, i. V.). Die regionalen Unterschiede, die in diesem Beitrag herausgearbeitet wurden, existieren jedoch unabhängig vom Faktor Alter.

Eine weiterführende Forschungsfrage, die in diesem Beitrag nicht verfolgt werden konnte, betrifft das Verhältnis zwischen grenzüberschreitender Identität und tatsächlich von den InformantInnen gesprochenem Dialekt. Betrachten wir das bereits eingangs erwähnte Beispiel des Kaiserstuhls. Die Analyse hat ergeben, dass für die Region Kaiserstuhl sowohl in der Selbst- als auch in der Fremdzuschreibung eine relativ starke Verbundenheit mit dem Elsass konstruiert wird, die in Bezug auf die geltend gemachten sprachlichen Gemeinsamkeiten besonders ausgeprägt ist. Diese Ergebnisse entsprechen der traditionellen dialektologischen Perspektive, die ebenfalls eine große Ähnlichkeit zwischen dem Dialekt des Kaiserstuhls und dem Elsässischen feststellt. Dieses auf den ersten Blick homogene Gesamtbild könnte nun durch das Einbeziehen aktueller dialektologischer Daten auf verschiedene Weise näher beleuchtet werden: Haben diejenigen Kaiserstühler Gewährspersonen, die eine gemeinsame Identität mit dem Elsass konstruieren, eine ältere Form ihres Ortsdialekts konserviert? Findet sich umgekehrt bei denjenigen Personen, die keine grenzüberschreitende Verbundenheit sehen, eine standardnähere Sprechweise? Falls solche Korrelationen nachweisbar sein sollten, wie sind sie zu interpretieren? Wäre es überhaupt plausibel, von einem kausalen Zusammenhang zwischen dem Vorhandensein einer grenzüberschreitenden Identität und starker Dialektalität auszugehen? Diesen und anderen Fragen, die sich analog auch für die anderen Regionen stellen, soll in zukünftiger Forschung nachgegangen werden, die sich einerseits auf die Quantifizierung 
der hier vorgestellten qualitativen Ergebnisse (cf. Pfeiffer, i. V.) und andererseits auf die dialektologischen Auswertungen (Spontansprache und Dialektkompetenz) des Projekts FLARS stützt.

\section{Literaturverzeichnis}

Abric, Jean-Claude (2016): Pratiques sociales et représentations. Paris: Presses Universitaires de France.

Auer, Peter (2018): „Das Beste zweier Welten: Das Bild elsässischer Dialektsprecher von den Deutschen, den Franzosen und sich selbst“". In: Lenz, Alexandra N./Plewnia, Albrecht (eds.): Variation - Normen - Identitäten. Berlin/Boston, de Gruyter: 5-40.

Auer, Peter/Breuninger, Julia/Pfeiffer, Martin (2017): „Neuere Entwicklungen des Alemannischen an der französisch-deutschen Sprachgrenze im Oberrheingebiet“. In: Christen, Helen/Gilles, Peter/Purschke, Christoph (eds.): Räume, Grenzen, Übergänge. Akten des 5. Kongresses der Internationalen Gesellschaft für Dialektologie des Deutschen (IGDD). Stuttgart, Steiner: 27-44. (= Zeitschrift für Dialektologie und Linguistik - Beihefte 171).

Auer, Peter et al. (2015): „Auswirkungen der Staatsgrenze auf die Sprachsituation im Oberrheingebiet (Frontière linguistique au Rhin Supérieur, FLARS)“. In: Kehrein, Roland/Lameli, Alfred/Rabanus, Stefan (eds.): Regionale Variation des Deutschen. Projekte und Perspektiven. Berlin/Boston, de Gruyter: 323-348.

Baldauf-Quilliatre, Heike (2016): ,' $p f^{\prime}$ indicating a change in orientation in French interaction“. Journal of Pragmatics 104: 89-107.

Bister-Broosen, Helga (1998): Sprachkontakte und Sprachattitüden Jugendlicher im Elsaß und in Baden. Vergleichende soziolinguistische Untersuchungen in Colmar (Frankreich) und in Freiburg und Müllheim (Deutschland). Frankfurt: Lang.

Bogatto, Francois-Xavier, et al. (2008): „Les représentations sociolinguistiques de locuteurs adolescents en Alsace (France)“. In Abel, Andrea/Stuflesser, Mathias/Putz, Magdalena (eds.): Tagungsband, Atti del Convegno, Proceedings, Mehrsprachigkeit in Europa, Plurilinguismo in Europa, Muliti-lingualism across Europe, 24-26.08.2006, Bolzano/Bozen. Bolzano, EURAC Research :89-100.

Bothorel-Witz, Arlette (2007): „Le plurilinguisme en Alsace: les représentations sociales comme resources de la description sociolinguistique“. Recherches en didactique des langues. L'Alsace au coeur du plurilinguisme (Les Cahiers de l'ACEDLE, 5, no 1): 41-63.

Breuninger, Julia (2016): „Die Frikativierung von intervokalisch -b- im Oberrheingebiet“. In: Ahner, Helen/Klausmann, Hubert (eds.): Dialekt und Öffentlichkeit. Beiträge zur 18. Arbeitstagung zur alemannischen Dialektologie. Tübingen. http://hdl.handle.net/10900/68024. [25.07.2019]

Bucholtz, Mary/Hall, Kira (2005): „Identity and interaction: a sociocultural linguistic approach“. Discourse Studies 7 (4-5): 585-614.

Busse, Hermann Eris (1939): „Volkstum“. In: Rest, Josef (ed.): Der Kaiserstuhl. Landschaft und Volkstum. Freiburg, Troemers Universitätsbuchhandlung: 253-266.

Erhart, Pascale (2019): „Von Staatsgrenze zu Dialektgrenze? Wird der Rhein als „Grenze“ im alemannischen Sprachraum am Oberrhein wahrgenommen?" Linguistik online 98, 5/19: 307-328. 
Fluck, Hans-Rüdiger (1974): Arbeit und Gerät im Wortschatz der Fischer des Hanauerlandes. Unteruchungen zur Fachsprache am Oberrhein. Freiburg/München: Alber.

Hansen-Morath, Sandra (2016): Regionale und soziolinguistische Variation im alemannischen Dreiländereck - Quantitative Studien zum Dialektwandel. Unveröff. Diss., Universität Freiburg.

Hoffmann, Rüdiger (1994): „Zur Sprachgeographie des Markgräflerlandes“. Das Markgräflerland 2/1994: 224-248. (= Jubiläumsband 550 Jahre Markgräflerland. Beiträge zu seiner Geschichte und Kultur).

Jodelet, Denise (1993): Les représentations sociales. Paris: Presses Universitaires de France.

Klausmann, Hubert (1990): „Staatsgrenze als Sprachgrenze? Zur Entstehung einer neuen Wortund Sprachgebrauchsgrenze am Oberrhein“. In: Kremer, Ludger/Niebaum, Hermann (eds.): Grenzdialekte. Studien zur Entwicklung kontinentalwestgermanischer Dialektkontinua. Hildesheim, Olms: 193-215.

Lucius-Hoene, Gabriele/Deppermann, Arnulf (2004): „Narrative Identität und Positionierung“. Gesprächsforschung - Online-Zeitschrift zur verbalen Interaktion 5: 166-183. www.gespraechsforschung-online.de/fileadmin/dateien/heft2004/ga-lucius.pdf [25.07.2019]

Mattheier, Klaus (1994): „Varietätenzensus. Über die Möglichkeiten, die Verbreitung und Verwendung von Sprachvarietäten in Deutschland festzustellen“. In: Mattheier, Klaus/Wiesinger, Peter (eds.): Dialektologie des Deutschen. Forschungsstand und Entwicklungstendenzen. Tübingen, Niemeyer: 413-442.

Maurer, Friedrich (1942): „Zur Sprachgeschichte des deutschen Südwestens“. In: Maurer, Friedrich (ed.): Oberrheiner, Schwaben, Südalemannen. Räume und Kräfte im geschichtlichen Aufbau des deutschen Südwestens. Straßburg, Hünenburg: 167-336.

Moscovici, Serge (2011): Psychologie sociale. Paris: Presses Universitaires de France.

Noth, Harald (1993): Alemannisches Dialekthandbuch vom Kaiserstuhl und seiner Umgebung. Freiburg: Schillinger.

Ochs, Ernst (1921): „Die Gliederung des Alemannischen“. Germanisch-romanische Monatsschrift 9: 56-58.

Ochs, Ernst (1939): „Mundart“. In: Rest, Josef (ed.): Der Kaiserstuhl. Landschaft und Volkstum. Freiburg, Troemers Universitätsbuchhandlung: 171-181.

Ochs, Ernst (1940): „Der oberrheinische Sprachraum“. In: Busse, Hermann Eris (ed.): Das Elsaß. Freiburg, Haus Badische Heimat: 431-432. (= Oberrheinische Heimat 27).

Pfeifer, Wolfgang (2005): Etymologisches Wörterbuch des Deutschen. 8. Auflage. München: Deutscher Taschenbuch Verlag.

Pfeiffer, Martin (i. V.): „Identität und Alterität an der französisch-deutschen Grenze im badischen Oberrheingebiet: Der Einfluss von Grenzentfernung, Wohnregion und Alter auf die wahrgenommene Verbundenheit mit dem Elsass“.

Pfeiffer, Martin/Auer, Peter (2019): „Erfahrung und Stereotyp an der elsässisch-badischen Grenze: Repräsentationen der Anderen und ihre narrative Verarbeitung“. In: Palliwoda, Nicole/Sauer, Verena/Sauermilch, Stephanie (eds.): Politische Grenzen - Sprachliche Grenzen? Dialektgeographische und wahrnehmungsdialektologische Perspektiven im deutschsprachigen Raum. Berlin/Boston: de Gruyter, 143-178. 
Rest, Josef (1939): „Mittelalterliche und neuere Geschichte“. In: Rest, Josef (ed.): Der Kaiserstuhl. Landschaft und Volkstum. Freiburg, Troemers Universitätsbuchhandlung: 87-118.

Rusch, Otto (1940): „Hanau-Lichtenberg. Eine Grafschaft zu beiden Seiten des Rheins“. In: Busse, Hermann Eris (ed.): Das Elsaß. Freiburg, Haus Badische Heimat: 197-205. (= Oberrheinische Heimat 27).

Schrambke, Renate (1981): Die sprachliche Stufenlandschaft am mittelbadischen Oberrhein. Diss., Freiburg i. Br.

Schrambke, Renate (2012): „Alemannisch zwischen Vogesen und Schwarzwald“. In: Homburger, Wolfgang et al. (eds.): Grenzüberschreitungen. Der alemannische Raum - Einheit trotz der Grenzen? Ostfildern, Thorbecke: 131-157.

Selting, Margret et al. (2009): „Gesprächsanalytisches Transkriptionssystem 2 (GAT 2) “. Gesprächsforschung - Online-Zeitschrift zur verbalen Interaktion 10: 152-183. www.gespraechsforschung-ozs.de/heft2009/px-gat2.pdf [25.07.2019]

Steger, Hugo et al. (1989-2011): Südwestdeutscher Sprachatlas (SSA). Marburg: Elwert.

Stoeckle, Philipp (2014): Subjektive Dialekträume im alemannischen Dreiländereck. Hildesheim: Olms. (= Deutsche Dialektgeographie 112).

Witzel, Andreas (1982): Verfahren der qualitativen Sozialforschung. Überblick und Alternativen. Frankfurt: Campus.

Witzel, Andreas (2000): „Das problemzentrierte Interview“. Forum Qualitative Sozialforschung 1 (1), Art. 22. DOI: http://dx.doi.org/10.17169/fqs-1.1.1132. [25.07.2019]

Wörner, Hans Jakob (1994): „Das Markgräflerland. Bemerkungen zu seinem geschichtlichen Werdegang“. Das Markgräflerland 2/1994: 56-69. (= Jubiläumsband 550 Jahre Markgräflerland. Beiträge zu seiner Geschichte und Kultur). 